Adv. Studies Theor. Phys., Vol. 8, 2014, no. 5, 215 - 249

HIKARI Ltd, www.m-hikari.com

http://dx.doi.org/10.12988/astp.2014.419

\title{
Higgs Mechanism in Scale-Invariant Gravity
}

\author{
Ichiro Oda \\ Department of Physics, Faculty of Science \\ University of the Ryukyus \\ Nishihara, Okinawa 903-0213, Japan
}

Copyright (C) 2014 Ichiro Oda. This is an open access article distributed under the Creative Commons Attribution License, which permits unrestricted use, distribution, and reproduction in any medium, provided the original work is properly cited.

\begin{abstract}
We consider a Higgs mechanism in scale-invariant theories of gravitation. It is shown that in spontaneous symmetry breakdown of scale invariance, gauge symmetries are also broken spontaneously even without the Higgs potential if the corresponding charged scalar fields couple to a scalar curvature in a non-minimal way. In this gravity-inspired new Higgs mechanism, the non-minimal coupling term, which is scaleinvariant, plays a critical role. Various generalizations of this mechanism are possible and particularly the generalizations to non-abelian gauge groups and a scalar field with multi-components are presented in some detail. Moreover, we apply our finding to a scale-invariant extension of the standard model (SM) and calculate radiative corrections. In particular, we elucidate the coupling between the dilaton and the Higgs particle and show that the dilaton mass takes a value around the $\mathrm{GeV}$ scale owing to quantum effects even if the dilaton is massless at the classical level.
\end{abstract}

\section{Introduction}

Current understanding of elementary particle physics is based on two celebrated fundamental principles, which are gauge symmetry and spontaneous symmetry breakdown of the gauge symmetry. In four interactions among elementary particles, strong, weak and electro-magnetic interactions are known to be described on the same footing in terms of a gauge theory which is the standard model $(\mathrm{SM})$ on the basis of $S U(3) \times S U(2) \times U(1)$ gauge group, and 
gravitational interaction is believed to be also described by a gauge theory whose final formalism is still far from complete at present.

The gauge principle alone, however, cannot describe the known structure of elementary particles. The gauge principle requires elementary particles to be massless ${ }^{1}$, so in order to generate masses for elementary particles the $S U(2) \times U(1)$ gauge symmetry must be spontaneously broken at any rate. The idea of spontaneous symmetry breakdown itself is not new for elementary particle physics but has emerged as a universal phenomenon in physics, in particular, condensed matter physics. An alternative and indeed older description of super-conductivity, which was developed by Ginzburg and Landau, turned out to be a phenomenological representation of the BCS theory [2]. In this transcription, the complex "Ginzburg-Landau" scalar field is nothing but the Higgs boson representing a bound pair of electrons and holes, and its phase and amplitude components correspond to the massless Nambu-Goldstone boson and the massive Higgs type of excitations, respectively. As it happens, the collective excitations of both the types do exist in all phenomena of the super-fluidity type. This universality of the spontaneous symmetry breakdown, however, seems to have no implication in gravity so far. The concept of the mass is intimately connected with general relativity since the right-hand side of Einstein's equations is constructed out of the energy-momentum tensor. In this article, we will investigate an idea such that a scale-invariant gravity induces the spontaneous symmetry breakdown of gauge symmetry without assuming the existence of the Higgs potential.

The SM based on $S U(3) \times S U(2) \times U(1)$ gauge group, together with classical general relativity, describes with amazing parsimony (only 19 parameters) our world over scales that have been explored by experiments: from the Hubble radius of $10^{30} \mathrm{~cm}$ all the way down to scales of the order of $10^{-16} \mathrm{~cm}$. In other words, with the help of cosmological initial conditions when the universe was much smaller, the SM is believed to encode the information needed to deduce all the physical phenomena observed so far. There are, however, some obvious chinks in the armor of the SM. In particular, the origin of different scales in nature cannot be answered at all by the SM. One should recall that there is only one fundamental constant with the dimension of mass: Gravity comes with its own mass scale $M_{p}=2.4 \times 10^{18} \mathrm{GeV}$. All units of mass should be scaled to this fundamental scale. It is a source of great intellectual worry that the SM appears to be consistent at a scale which is so different from the Planck mass scale. Naive expectations are that all physical phenomena should occur

\footnotetext{
${ }^{1}$ To tell this statement more precisely, gauge invariance with the conventional form forbids the presence of a mass term of gauge field, but such a mass term can exist if we change the gauge transformation to a more complicated form. This situation happens when we consider a theory with spontaneous symmetry beakdown where a translation of the field by a constant must be accompanied [1].
} 
at their natural scale which is of course the Planck scale.

Coleman-Mandula theorem [3] allows the Poincare group to be generalized to two global groups, one is the super-Poincare group and the other is the conformal group. It is remarkable to notice that these two groups might yield resolution of the gauge hierarchy problem by a completely different idea, and they also yield a natural generalization of local gauge group, the former gives rise to the local super-Poincare group leading to supergravity whereas the latter does the local conformal group leading to conformal gravity.

According to recent results by the LHC $[4,5]$, supersymmetry on the basis of the super-Poincare group seems not to be taken by nature as resolution of the gauge hierarchy problem. Then, it is natural to ask ourselves if the conformal group, the other extension of the Poincare group, gives us resolution of the gauge hierarchy problem. Indeed, inspired by an interesting idea by Bardeen [6], there has appeared to pursue the possibility of replacing the supersymmetry with the conformal symmetry near the $\mathrm{TeV}$ scale in an attempt to solve the hierarchy problem $[7,8]$. It is worth noting that the principle of conformal invariance is more rigid than the supersymmetry in the sense that in many examples the conformal symmetry predicts the number of generations as well as a rich structure for the Yukawa couplings among various families. This inter-family rigidity is a welcome feature of the conformal approach to particle phenomenology [9].

In the conformal approach, it is thought that the electro-weak scale and the QCD scale as well as the masses of observed quarks and leptons are all so small compared to the Planck scale that it is reasonable to believe that in some approximation they are exactly massless. If so, then the quantum field theory which would be describing the massless fields should be a conformal theory as it has no mass scale. In this scenario, the fact that there are no large mass corrections follows from the condition of conformal invariance. In other words, the 'tHooft naturalness condition [10] is satisfied in the conformal approach, namely in the absence of masses there is an enhanced symmetry which is the conformal symmetry. Of course, the breaking of conformal invariance should be soft in such a way that the idea of the conformal symmetry is relevant for solving the hierarchy problem.

In passing, in the present context, it seems to be of interest to consider the issue of renormalizability. Usually, in quantum field theories, the condition of renormalizability is imposed on a theory as if it were a basic principle to make the perturbation method to be meaningful, but its real meaning is unclear since there might exist a theory for which only the non-perturbative approach could be applied without relying on the perturbation method at all. To put differently, the concept of renormalizability means that even if one is unfamiliar with true physics beyond some higher energy scale, one can construct an effective theory by confining its ignorance to some parameters such as coupling 
constants and masses below the energy scale. Thus, from this point of view, it is unclear to require the renormalizability to theories holding at the highest energy scale, the Planck scale, such as quantum gravity and superstring theory. On the other hand, given a scale invariance in a theory, all the coupling constants must be dimensionless and operators in an action are marginal ones whose coefficient is independent of a certain scale, which ensures that the theory is manifestly renormalizable. In this world, all masses of particles must be then generated by spontaneous symmetry breakdown.

In previous works $[11,12]$, we have shown that without resort to the Coleman-Weinberg mechanism [13], by coupling the non-minimal term of gravity, the $\mathrm{U}(1)$ B-L gauge symmetry in the model [8] is spontaneously broken in the process of spontaneous symmetry breakdown of global or local scale symmetry at the tree level and as a result the U(1) B-L gauge field becomes massive via the Higgs mechanism. One of advantages in this mechanism is that we do not have to introduce the Higgs potential in a theory.

Then, we have the following questions of this mechanism of symmetry breaking of gauge symmetry:

1. Is it possible to generalize to the non-abelian gauge groups?

2. Is it possible to generalize many scalar fields?

3. What becomes of applying it to the standard model and what its radiative corrections are?

In this article, we would like to answer these questions in order. The structure of this article is the following: In Section 2, we present the simplest model which accomodates global scale symmetry and the abelian gauge symmetry, and explain our main idea. In Section 3, we generalize this simple model to a model with the non-abelian gauge symmetry. In Section 4, we extend our idea to a model of a scalar field with many of components. Moreover, we apply our finding to a scale-invariant extension of the standard model and calculate radiative corrections in Section 5. We conclude in Section 6. Two appendices are given, one of which is to explain a derivation of the dilatation current and the other is to put useful formulae for the calcualtion of radiative corrections.

\section{Review of a globally scale-invariant Abelian model}

We start with a brief review of the simplest model showing a gravitational Higgs phenomenon which was previously discovered in case of a global scale invariance and the abelian gauge group [11]. 
With a background curved metric $g_{\mu \nu}$, a complex (singlet) scalar field $\Phi$ and the $U(1)$ gauge field $A_{\mu}$, the Lagrangian takes the form ${ }^{2}$ :

$$
\mathcal{L}=\sqrt{-g}\left[\xi \Phi^{\dagger} \Phi R-g^{\mu \nu}\left(D_{\mu} \Phi\right)^{\dagger}\left(D_{\nu} \Phi\right)-\frac{1}{4} g^{\mu \nu} g^{\rho \sigma} F_{\mu \rho} F_{\nu \sigma}\right]
$$

where $\xi$ is a certain positive and dimensionless constant. The covariant derivative and field strength are respectively defined as

$$
D_{\mu} \Phi=\left(\partial_{\mu}-i e A_{\mu}\right) \Phi, \quad\left(D_{\mu} \Phi\right)^{\dagger}=\left(\partial_{\mu}+i e A_{\mu}\right) \Phi^{\dagger}, \quad F_{\mu \nu}=\partial_{\mu} A_{\nu}-\partial_{\nu} A_{\mu}
$$

with $e$ being a $U(1)$ real coupling constant.

Let us note that the Lagrangian $(1)$ is invariant under a global scale transformation. In fact, with a constant parameter $\Omega=e^{\Lambda} \approx 1+\Lambda(|\Lambda| \ll 1)$ the scale transformation is defined as $[15]^{3}$

$$
\begin{aligned}
g_{\mu \nu} & \rightarrow \tilde{g}_{\mu \nu}=\Omega^{2} g_{\mu \nu}, \quad g^{\mu \nu} \rightarrow \tilde{g}^{\mu \nu}=\Omega^{-2} g^{\mu \nu} \\
\Phi & \rightarrow \tilde{\Phi}=\Omega^{-1} \Phi, \quad A_{\mu} \rightarrow \tilde{A}_{\mu}=A_{\mu} .
\end{aligned}
$$

Then, using the formulae $\sqrt{-g}=\Omega^{-4} \sqrt{-\tilde{g}}, R=\Omega^{2} \tilde{R}$, it is straightforward to show that $\mathcal{L}$ is invariant under the scale transformation (3). Following the Noether procedure $\Lambda J^{\mu}=\sum \frac{\partial \mathcal{L}}{\partial \partial_{\mu} \phi} \delta \phi$ where $\phi=\left\{g_{\mu \nu}, \Phi, \Phi^{\dagger}\right\}$, as shown in the Appendix A, the current for the scale transformation, what we call the dilatation current, takes the form ${ }^{4}$

$$
J^{\mu}=(6 \xi+1) \sqrt{-g} g^{\mu \nu} \partial_{\nu}\left(\Phi^{\dagger} \Phi\right) .
$$

To prove that this current is conserved on-shell, it is necessary to derive a set of equations of motion from the Lagrangian (1). The variation of (1) with respect to the metric tensor produces Einstein's equations

$$
2 \xi \Phi^{\dagger} \Phi G_{\mu \nu}=T_{\mu \nu}^{(A)}+T_{\mu \nu}^{(\Phi)}-2 \xi\left(g_{\mu \nu} \square-\nabla_{\mu} \nabla_{\nu}\right)\left(\Phi^{\dagger} \Phi\right)
$$

\footnotetext{
${ }^{2}$ We follow notation and conventions by Misner et al.'s textbook [14], for instance, the flat Minkowski metric $\eta_{\mu \nu}=\operatorname{diag}(-,+,+,+)$, the Riemann curvature tensor $R^{\mu}{ }_{\nu \alpha \beta}=$ $\partial_{\alpha} \Gamma_{\nu \beta}^{\mu}-\partial_{\beta} \Gamma_{\nu \alpha}^{\mu}+\Gamma_{\sigma \alpha}^{\mu} \Gamma_{\nu \beta}^{\sigma}-\Gamma_{\sigma \beta}^{\mu} \Gamma_{\nu \alpha}^{\sigma}$, and the Ricci tensor $R_{\mu \nu}=R_{\mu \alpha \nu}^{\alpha}$. The reduced Planck mass is defined as $M_{p}=\sqrt{\frac{c \hbar}{8 \pi G}}=2.4 \times 10^{18} \mathrm{GeV}$. Through this article, we adopt the reduced Planck units where we set $c=\hbar=M_{p}=1$ though we sometimes recover the Planck mass $M_{p}$ for the clarification of explanation. In this units, all quantities become dimensionless. Finally, note that in the reduced Planck units, the Einstein-Hilbert Lagrangian density takes the form $\mathcal{L}_{E H}=\frac{1}{2} \sqrt{-g} R$.

${ }^{3}$ In this article, we use the terminology such that scale or conformal transformation means global transformation whereas its local version is called local scale transformation or local conformal transformation.

${ }^{4}$ The case $\xi=-\frac{1}{6}$ corresponds to conformal gravity, for which there is no dilatation current.
} 
where d'Alembert operator $\square$ is as usual defined as $\square\left(\Phi^{\dagger} \Phi\right)=\frac{1}{\sqrt{-g}} \partial_{\mu}\left(\sqrt{-g} g^{\mu \nu} \partial_{\nu}\left(\Phi^{\dagger} \Phi\right)\right)=$ $g^{\mu \nu} \nabla_{\mu} \nabla_{\nu}\left(\Phi^{\dagger} \Phi\right)$ and the Einstein tensor is $G_{\mu \nu}=R_{\mu \nu}-\frac{1}{2} g_{\mu \nu} R$. Here the energymomentum tensors $T_{\mu \nu}^{(A)}$ for the gauge field and $T_{\mu \nu}^{(\Phi)}$ for the scalar field are defined as, respectively

$$
\begin{aligned}
T_{\mu \nu}^{(A)} & =-\frac{2}{\sqrt{-g}} \frac{\delta}{\delta g^{\mu \nu}}\left[-\frac{1}{4} \sqrt{-g} g^{\alpha \beta} g^{\rho \sigma} F_{\alpha \rho} F_{\beta \sigma}\right] \\
& =g^{\rho \sigma} F_{\mu \rho} F_{\nu \sigma}-\frac{1}{4} g_{\mu \nu} F_{\rho \sigma}^{2}, \\
T_{\mu \nu}^{(\Phi)} & =-\frac{2}{\sqrt{-g}} \frac{\delta}{\delta g^{\mu \nu}}\left[-\sqrt{-g} g^{\rho \sigma}\left(D_{\rho} \Phi\right)^{\dagger}\left(D_{\sigma} \Phi\right)\right] \\
& =2\left(D_{(\mu} \Phi\right)^{\dagger}\left(D_{\nu)} \Phi\right)-g_{\mu \nu}\left(D_{\rho} \Phi\right)^{\dagger}\left(D^{\rho} \Phi\right),
\end{aligned}
$$

where we have used the notation of symmetrization $A_{(\mu} B_{\mu)}=\frac{1}{2}\left(A_{\mu} B_{\nu}+A_{\nu} B_{\mu}\right)$.

Next, the equation of motion for $\Phi^{\dagger}$ is of form

$$
\xi \Phi R+\frac{1}{\sqrt{-g}} D_{\mu}\left(\sqrt{-g} g^{\mu \nu} D_{\nu} \Phi\right)=0
$$

Finally, taking the variation with respect to the gauge fields $A_{\mu}^{(i)}$ produces "Maxwell" equations

$$
\nabla_{\rho} F^{\mu \rho}=-i e\left[\Phi^{\dagger}\left(D^{\mu} \Phi\right)-\Phi\left(D^{\mu} \Phi\right)^{\dagger}\right]
$$

Now we wish to prove that the current (4) for the scale transformation is indeed conserved on-shell by using these equations of motion. Before doing so, let us first take the divergence of the current, whose result is given by

$$
\partial_{\mu} J^{\mu}=(6 \xi+1) \sqrt{-g} \square\left(\Phi^{\dagger} \Phi\right) .
$$

In order to show that the expression in the right-hand side of Eq. (9) vanishes on-shell, let us take the trace of Einstein's equations (5)

$$
\xi \Phi^{\dagger} \Phi R=3 \xi \square\left(\Phi^{\dagger} \Phi\right)+\left(D_{\mu} \Phi\right)^{\dagger}\left(D^{\mu} \Phi\right) .
$$

Next, multiplying Eq. (7) by $\Phi^{\dagger}$, and then eliminating the term involving the scalar curvature, i.e., $\xi \Phi^{\dagger} \Phi R$, with the help of Eq. (10), we obtain

$$
3 \xi \square\left(\Phi^{\dagger} \Phi\right)+\left(D_{\mu} \Phi\right)^{\dagger}\left(D^{\mu} \Phi\right)+\frac{1}{\sqrt{-g}} \Phi^{\dagger} D_{\mu}\left(\sqrt{-g} g^{\mu \nu} D_{\nu} \Phi\right)=0 .
$$

At this stage, it is useful to introduce a generalized covariant derivative defined as $\mathcal{D}_{\mu}=D_{\mu}+\Gamma_{\mu}$ where $\Gamma_{\mu}$ is the usual affine connection. Using this derivative, Eq. (11) can be rewritten as

$$
3 \xi \mathcal{D}_{\mu} \mathcal{D}^{\mu}\left(\Phi^{\dagger} \Phi\right)+\left(\mathcal{D}_{\mu} \Phi\right)^{\dagger}\left(\mathcal{D}^{\mu} \Phi\right)+\Phi^{\dagger} \mathcal{D}_{\mu} \mathcal{D}^{\mu} \Phi=0
$$


Then, adding its Hermitian conjugation to Eq. (12), we arrive at

$$
(6 \xi+1) \mathcal{D}_{\mu} \mathcal{D}^{\mu}\left(\Phi^{\dagger} \Phi\right)=0
$$

The quantity $\Phi^{\dagger} \Phi$ is a scalar and neutral under the $U(1)$ charge, we obtain

$$
(6 \xi+1) \square\left(\Phi^{\dagger} \Phi\right)=0
$$

Using this equation, the right-hand side in Eq. (9) is certainly vanishing, by which we can prove that the current of the scale transformation is conserved on-shell as promised.

Now we are willing to explain our finding about spontaneous symmetry breakdown of gauge symmetry in our model where the coexistence of both scale invariance and gauge symmetry plays a pivotal role. Incidentally, it might be worthwhile to comment that in ordinary examples of spontaneous symmetry breakdown in the framework of quantum field theories, one is accustomed to dealing with a potential which has the shape of the Mexican hat type and therefore induces the symmetry breaking in a natural way, but the same recipe cannot be applied to general relativity because of the lack of such a potential. ${ }^{5}$

Let us note that a very interesting recipe which induces spontaneous symmetry breakdown of scale invariance via local scale transformation has been already known [15]. This recipe can be explained as follows: Suppose that we started with a scale-invariant theory with only dimensionless coupling constants. But in the process of local scale transformation, one cannot refrain from introducing the quantity with mass dimension, which is the Planck mass $M_{p}$ in the present context, to match the dimensions of an equation and consequently scale invariance is spontaneously broken.

Of course, the absence of a potential which induces symmetry breaking makes it impossible to investigate a stability of the selected solution, but the very existence of the solution including the Planck mass with mass dimension justifies the claim that this phenomenon is nothing but a sort of spontaneous symmetry breakdown. This fact can be also understood by using a dilatation charge as seen shortly.

The first technique for obtaining spontaneous symmetry breakdown of both scale and gauge invariances is to find a suitable local scale transformation which transforms dilaton gravity in the Jordan frame to general relativity with matters in the Einstein frame. Of course, note that our starting Lagrangian is invariant under not the local scale transformation but the global transformation, so the change of form of the Lagrangian after the local scale transformation is reasonable. Here it is useful to parametrize the complex scalar field $\Phi$ in terms

\footnotetext{
${ }^{5}$ In the case of massive gravity, a similar situation occurs in breaking the general coordinate invariance spontaneously [16].
} 
of two real fields, $\Omega$ (or $\sigma$ ) and $\theta$ in polar form, defined as

$$
\Phi(x)=\frac{1}{\sqrt{2 \xi}} \Omega(x) e^{i \alpha \theta(x)}=\frac{1}{\sqrt{2 \xi}} e^{\zeta \sigma(x)+i \alpha \theta(x)},
$$

where $\Omega(x)=e^{\zeta \sigma(x)}$ is a local parameter field and the constants $\zeta, \alpha$ will be determined later.

Let us then consider the following local scale transformation:

$$
g_{\mu \nu} \rightarrow \tilde{g}_{\mu \nu}=\Omega^{2}(x) g_{\mu \nu}, \quad g^{\mu \nu} \rightarrow \tilde{g}^{\mu \nu}=\Omega^{-2}(x) g^{\mu \nu}, \quad A_{\mu} \rightarrow \tilde{A}_{\mu}=A_{\mu} .
$$

Note that apart from the local property of $\Omega(x)$, this local scale transformation is different from the scale transformation (3) in that the complex scalar field $\Phi$ is not transformed at all. Under the local scale transformation (16), the scalar curvature is transformed as

$$
R=\Omega^{2}\left(\tilde{R}+6 \square f-6 \tilde{g}^{\mu \nu} \partial_{\mu} f \partial_{\nu} f\right),
$$

where we have defined as $f=\log \Omega=\zeta \sigma$ and $\square f=\frac{1}{\sqrt{-\tilde{g}}} \partial_{\mu}\left(\sqrt{-\tilde{g}} \tilde{g}^{\mu \nu} \partial_{\nu} f\right)=$ $\tilde{g}^{\mu \nu} \tilde{\nabla}_{\mu} \tilde{\nabla}_{\nu} f$.

With the critical choice

$$
\xi \Phi^{\dagger} \Phi=\frac{1}{2} \Omega^{2}=\frac{1}{2} e^{2 \zeta \sigma}
$$

the non-minimal term in (1) reads the Einstein-Hilbert term (plus part of the kinetic term of the scalar field $\sigma$ ) up to a surface term as follows:

$$
\begin{aligned}
\sqrt{-g} \xi \Phi^{\dagger} \Phi R & =\Omega^{-4} \sqrt{-\tilde{g}} \frac{1}{2} \Omega^{2} \Omega^{2}\left(\tilde{R}+6 \square f-6 \tilde{g}^{\mu \nu} \partial_{\mu} f \partial_{\nu} f\right) \\
& =\sqrt{-\tilde{g}}\left(\frac{1}{2} \tilde{R}-3 \zeta^{2} \tilde{g}^{\mu \nu} \partial_{\mu} \sigma \partial_{\nu} \sigma\right)
\end{aligned}
$$

Then, the second term in (1) is cast to the form

$$
-\sqrt{-g} g^{\mu \nu}\left(D_{\mu} \Phi\right)^{\dagger}\left(D_{\nu} \Phi\right)=-\frac{1}{2 \xi} \sqrt{-\tilde{g}} \tilde{g}^{\mu \nu}\left(\zeta^{2} \partial_{\mu} \sigma \partial_{\nu} \sigma+e^{2} B_{\mu} B_{\nu}\right)
$$

where we have chosen $\alpha=e$ for convenience, and defined a new massive gauge field $B_{\mu}$ as

$$
B_{\mu}=A_{\mu}+\partial_{\mu} \theta \text {. }
$$

In terms of this new gauge field $B_{\mu}$, the Maxwell's Lagrangian in (1) is described in the Einstein frame as

$$
-\frac{1}{4} \sqrt{-g} g^{\mu \nu} g^{\rho \sigma} F_{\mu \rho} F_{\nu \sigma}=-\frac{1}{4} \sqrt{-\tilde{g}} \tilde{g}^{\mu \nu} \tilde{g}^{\rho \sigma} \tilde{F}_{\mu \rho} \tilde{F}_{\nu \sigma}
$$


where $\tilde{F}_{\mu \nu} \equiv \partial_{\mu} B_{\nu}-\partial_{\nu} B_{\mu}$.

It is worthwhile to stress again that in the process of local scale transformation we have had to introduce the mass scale into a theory having no dimensional constants, thereby inducing the breaking of the scale invariance. More concretely, to match the dimensions in the both sides of the equation, the Planck mass $M_{p}$ must be introduced in the ciritical choice (18) (recovering the Planck mass)

$$
\xi \Phi^{\dagger} \Phi=\frac{1}{2} \Omega^{2} M_{p}^{2}=\frac{1}{2} e^{2 \zeta \sigma} M_{p}^{2}
$$

It is also remarkable to notice that in the process of spontaneous symmetry breakdown of the scale invariance, the Nambu-Goldstone boson $\theta$ is absorbed into the $U(1)$ gauge field $A_{\mu}$ as a longitudinal mode and as a result $B_{\mu}$ acquires a mass, which is nothing but the Higgs mechanism! In other words, the $U(1)$ gauge symmetry is broken at the same time and on the same energy scale that the scale symmetry is spontaneously broken. The size of the mass $M_{B}$ of $B_{\mu}$ can be read off from (20) as $M_{B}=\frac{e}{\sqrt{\xi}} M_{p}$ which is also equal to the energy scale on which the scale invariance is broken.

Putting (19), (20) and (22) together, and defining $\zeta^{-2}=6+\frac{1}{\xi}$ (by which the kinetic term for the $\sigma$ field becomes a canonical form), the Lagrangian (1) is reduced to the form

$$
\mathcal{L}=\sqrt{-\tilde{g}}\left[\frac{1}{2} M_{p}^{2} \tilde{R}-\frac{1}{2} \tilde{g}^{\mu \nu} \partial_{\mu} \sigma \partial_{\nu} \sigma-\frac{1}{4} \tilde{F}_{\mu \nu}^{2}-\frac{e^{2}}{2 \xi} M_{p}^{2} B_{\mu} B^{\mu}\right],
$$

where we have recovered the Planck mass $M_{p}$ for clarity. Let us note that the first term coincides with the Einstein-Hilbert term in general relativity, the second term implies that the dilaton $\sigma$ is massless at the classical level, and the last two terms means that the gauge field becomes massive via the new Higgs mechanism.

As an interesting application of our finding to phenomenology, we can propose two scenarios at the different energy scales. One scenario, which was adopted in case of the classically scale-invariant B-L model $[8,11,12]$, is the spontaneous symmetry breakdown at the TeV scale where $\frac{e}{\sqrt{\xi}} \approx 10^{-15}$, so the gravity is in the strong coupling phase. The other scenario is to trigger the spontaneous symmetry breakdown of both scale and gauge symmetry at the Planck scale, for which we take $\frac{e}{\sqrt{\xi}} \approx 1$ and the gravity is in the weak coupling phase.

Finally, let us comment on the physical meaning of the dilaton $\sigma$. The dilaton is a massless particle and interact with the other fields only through the covariant derivative $\tilde{D}_{\mu}=D_{\mu}+\zeta\left(\partial_{\mu} \sigma\right)$, but owing to its nature of the derivative coupling, at the low energy this coupling is so small that it is difficult to detect the dilaton experimentally. 
To understand the physical meaning of the dilaton more clearly, it is useful to evaluate the dilatation current $J^{\mu}$ in (4) in the Einstein frame. The result reads

$$
J^{\mu}=\frac{1}{\zeta} \sqrt{-\tilde{g}} \tilde{g}^{\mu \nu} \partial_{\nu} \sigma
$$

This is exactly the expected form of the current seen in the case of the conventional spontaneous symmetry breakdown, with $\frac{1}{\zeta}$ playing the role of the vacuum value of the order parameter, and the dilaton $\sigma$ doing of the NambuGoldstone boson associated with the spontaneous symmetry breakdown of the scale invariance. This result can be also reached by constructing the corresponding charge which is defined as $Q_{D}=\int d^{3} x J^{0}$. Note that this charge does not annihilate the vacuum because of the linear form in $\sigma$

$$
Q_{D} \mid 0>\neq 0
$$

Of course, it is also possible to show $\partial_{\mu} J^{\mu}=0$ in terms of equations of motion in the Einstein frame as proved in the Jordan frame before. It therefore turns out that the dilaton $\sigma$ is indeed the Nambu-Goldstone boson associated with spontaneous symmetry breakdown of the scale invariance. We will see later that although the dilaton is massless at the classical level, the trace anomaly makes the dilaton be massive at the quantum level.

\section{The generalization to non-Abelian groups}

In this section, we wish to extend the present formalism to arbitrary nonAbelian gauge groups. For clarity, we shall consider only the $S U(2)$ gauge group with a complex $S U(2)$-doublet of scalar field $\Phi^{T}=\left(\Phi_{1}, \Phi_{2}\right)$ since the generalization to a general non-Abelian gauge group is straightforward.

Let us start with the $S U(2)$ generalization of the Lagrangian (1)

$$
\mathcal{L}=\sqrt{-g}\left[\xi \Phi^{\dagger} \Phi R-g^{\mu \nu}\left(D_{\mu} \Phi\right)^{\dagger}\left(D_{\nu} \Phi\right)-\frac{1}{4} g^{\mu \nu} g^{\rho \sigma} F_{\mu \rho}^{a} F_{\nu \sigma}^{a}\right]
$$

where $a$ is an $S U(2)$ index running over 1,2,3, and the covariant derivative and field strength are respectively defined as

$$
\begin{aligned}
D_{\mu} \Phi & =\left(\partial_{\mu}-i g \tau^{a} A_{\mu}^{a}\right) \Phi, \quad\left(D_{\mu} \Phi\right)^{\dagger}=\left(\partial_{\mu}+i g \tau^{a} A_{\mu}^{a}\right) \Phi^{\dagger} \\
F_{\mu \nu}^{a} & =\partial_{\mu} A_{\nu}^{a}-\partial_{\nu} A_{\mu}^{a}+g \varepsilon^{a b c} A_{\mu}^{b} A_{\nu}^{c} .
\end{aligned}
$$

Here $g$ is an $S U(2)$ coupling constant (Do not confuse with the determinant of the metric tensor since we use the same letter of the alphabet). Furthermore, 
the matrices $\tau^{a}$ are defined as half of the Pauli ones, i.e., $\tau^{a}=\frac{1}{2} \sigma^{a}$, so the following relations are satisfied:

$$
\left\{\tau^{a}, \tau^{b}\right\}=\frac{1}{2} \delta^{a b}, \quad\left[\tau^{a}, \tau^{b}\right]=i \varepsilon^{a b c} \tau^{c} .
$$

In order to see the Higgs mechanism discussed in the previous section explicitly, it is convenient to go to the unitary gauge. To do that, we first parametrize the scalar doublet as

$$
\Phi(x)=U^{-1}(x) \frac{1}{\sqrt{2 \xi}} e^{\zeta \sigma(x)}\left(\begin{array}{l}
0 \\
1
\end{array}\right)
$$

where a unitary matrix $U(x)$ is defined as $U(x)=e^{-i \alpha \tau^{a} \theta^{a}(x)}$ with $\alpha$ being a real number. Then, we will define new fields in the unitary gauge by

$$
\begin{aligned}
\Phi^{u}(x) & =U(x) \Phi(x)=\frac{1}{\sqrt{2 \xi}} e^{\zeta \sigma(x)}\left(\begin{array}{l}
0 \\
1
\end{array}\right)=\frac{1}{\sqrt{2 \xi}} \Omega(x)\left(\begin{array}{l}
0 \\
1
\end{array}\right), \\
\tau^{a} B_{\mu}^{a} & =U(x) \tau^{a} A_{\mu}^{a} U^{-1}(x)-\frac{i}{g} \partial_{\mu} U(x) U^{-1}(x) .
\end{aligned}
$$

Using these new fields, after an easy calculation, we find the following relations

$$
D_{\mu} \Phi=U^{-1}(x) D_{\mu} \Phi^{u}, \quad F_{\mu \nu}^{a} F^{a \mu \nu}=F_{\mu \nu}^{a}(B) F^{a \mu \nu}(B),
$$

where $D_{\mu} \Phi^{u}$ and $F_{\mu \nu}^{a}(B)$ are respectively defined as

$$
D_{\mu} \Phi^{u}=\left(\partial_{\mu}-i g \tau^{a} B_{\mu}^{a}\right) \Phi^{u}, \quad F_{\mu \nu}^{a}(B)=\partial_{\mu} B_{\nu}^{a}-\partial_{\nu} B_{\mu}^{a}+g \varepsilon^{a b c} B_{\mu}^{b} B_{\nu}^{c} .
$$

To reach the desired Lagrangian, we can follow a perfectly similar path of argument to the case of the Abelian gauge group in the previous section. In other words, we will take a critical choice

$$
\xi \Phi^{u \dagger} \Phi^{u}=\frac{1}{2} \Omega^{2} M_{p}^{2}=\frac{1}{2} e^{2 \zeta \sigma} M_{p}^{2}
$$

and make use of the local scale transformation by the local parameter $\Omega(x)$ to move from the Jordan frame to the Einstein frame. After performing this procedure, the final Lagrangian reads

$$
\mathcal{L}=\sqrt{-\tilde{g}}\left[\frac{1}{2} M_{p}^{2} \tilde{R}-\frac{1}{2} \tilde{g}^{\mu \nu} \partial_{\mu} \sigma \partial_{\nu} \sigma-\frac{1}{4}\left(\tilde{F}_{\mu \nu}^{a}\right)^{2}-\frac{g^{2}}{8 \xi} M_{p}^{2} B_{\mu}^{a} B^{a \mu}\right]
$$

where $\tilde{F}_{\mu \nu}^{a} \equiv F_{\mu \nu}^{a}(B)$. The mass of the massive gauge field $B_{\mu \nu}^{a}$ is easily read off to be $M_{B}=\frac{g}{2 \sqrt{\xi}} M_{p}$. As in the Abelian group, we can see that the massless dilaton $\sigma$ is the Nambu-Goldstone boson of spontaneous symmetry breakdown of scale symmetry by making the conserved dilatation current and its charge. 


\section{The generalization to scalar field with many components}

Let us recall that the quantum field theory of a scalar field with many components goes in much the same way as that of a single component except that new interesting internal symmetry arises. This general fact is also valid even in the present formalism if we take a common "radial" field in all the components. When we consider a general "radial" field, we must face an annoying issue of getting the canonical kinetic term for the dilaton. The procedure of obtaining the canonical kinetic term is just a problem of matrix diagonalization and is not in principle a problem, but the general treatment makes our formalism very complicated. We will therefore focus on the case of the common "radial" field in this section. In the next section, we will meet the same situation since we consider two scalar fields coupling to a curvature scalar in the non-minimal manner, but a reasonable approximation can serve to avoid this annoying issue.

The starting Lagrangian is just a generalization of (1) to $n$ complex scalar fields, or equivalently a complex scalar field with $n$ independent components $\Phi_{i}(i=1,2, \cdots, n)$

$$
\mathcal{L}=\sum_{i=1}^{n} \sqrt{-g}\left[\xi_{i} \Phi_{i}^{\dagger} \Phi_{i} R-g^{\mu \nu}\left(D_{\mu} \Phi_{i}\right)^{\dagger}\left(D_{\nu} \Phi_{i}\right)-\frac{1}{4} g^{\mu \nu} g^{\rho \sigma} F_{\mu \rho}^{(i)} F_{\nu \sigma}^{(i)}\right],
$$

where $\xi_{i}$ are positive and dimensionless constants. The covariant derivative and field strength are respectively defined as

$D_{\mu} \Phi_{i}=\left(\partial_{\mu}-i e_{i} A_{\mu}^{(i)}\right) \Phi_{i}, \quad\left(D_{\mu} \Phi_{i}\right)^{\dagger}=\left(\partial_{\mu}+i e_{i} A_{\mu}^{(i)}\right) \Phi_{i}^{\dagger}, \quad F_{\mu \nu}^{(i)}=\partial_{\mu} A_{\nu}^{(i)}-\partial_{\nu} A_{\mu}^{(i)}$

Since the Lagrangian (36) includes only dimensionless coupling constants, it is manifestly invariant under a global scale transformation. Following the Noether theorem, the current for the scale transformation reads

$$
J^{\mu}=\sum_{i=1}^{n}\left(6 \xi_{i}+1\right) \sqrt{-g} g^{\mu \nu} \partial_{\nu}\left(\Phi_{i}^{\dagger} \Phi_{i}\right) .
$$

In a similar way to the cases of both Abelian and non-Abelian gauge groups, we can show that this current is conserved on-shell.

As mentioned in the above, the point is to take a common "radial" (real) field $\Omega(x)$ such that

$$
\Phi_{i}(x)=\frac{1}{\sqrt{2 n \xi_{i}}} \Omega(x) e^{i \alpha_{i} \theta_{i}(x)}=\frac{1}{\sqrt{2 n \xi_{i}}} e^{\zeta \sigma(x)+i \alpha_{i} \theta_{i}(x)} .
$$

This is a great simplification in the sense that $n$ real component fields in $\Phi_{i}(x)$ is reduced to a single one, but this restriction is needed to obtain the canonical kinetic term for the dilaton in a rather simple way. 
Now we would like to show that the Lagrangian (36) has the property of spontaneous symmetry breakdown of gauge symmetry when scale symmetry is spontaneously broken. To do that, we proceed similar steps to the case of the single scalar field with the Abelian gauge group in Section 2. With the critical choice

$$
\xi_{i} \Phi_{i}^{\dagger} \Phi_{i}=\frac{1}{2 n} \Omega^{2}=\frac{1}{2 n} e^{2 \zeta \sigma}
$$

the non-minimal term in (36) yields the Einstein-Hilbert term and part of the kinetic term of the scalar field $\sigma$ ) up to a surface term

$$
\sum_{i=1}^{n} \sqrt{-g} \xi_{i} \Phi_{i}^{\dagger} \Phi_{i} R=\sqrt{-\tilde{g}}\left(\frac{1}{2} \tilde{R}-3 \zeta^{2} \tilde{g}^{\mu \nu} \partial_{\mu} \sigma \partial_{\nu} \sigma\right)
$$

Moreover, the second term in (36) is reduced to

$-\sum_{i=1}^{n} \sqrt{-g} g^{\mu \nu}\left(D_{\mu} \Phi_{i}\right)^{\dagger}\left(D_{\nu} \Phi_{i}\right)=-\sum_{i=1}^{n} \frac{1}{2 n \xi_{i}} \sqrt{-\tilde{g}} \tilde{g}^{\mu \nu}\left(\zeta^{2} \partial_{\mu} \sigma \partial_{\nu} \sigma+e_{i}^{2} B_{\mu}^{(i)} B_{\nu}^{(i)}\right)$

where we have selected $\alpha_{i}=e_{i}$ and defined new massive gauge fields $B_{\mu}^{(i)}$ by

$$
B_{\mu}^{(i)}=A_{\mu}^{(i)}+\partial_{\mu} \theta_{i}
$$

In terms of the new gauge fields $B_{\mu}^{(i)}$, the Maxwell's Lagrangian in (36) is cast to the form

$$
-\frac{1}{4} \sum_{i=1}^{n} \sqrt{-g} g^{\mu \nu} g^{\rho \sigma} F_{\mu \rho}^{(i)} F_{\nu \sigma}^{(i)}=-\frac{1}{4} \sum_{i=1}^{n} \sqrt{-\tilde{g}} \tilde{g}^{\mu \nu} \tilde{g}^{\rho \sigma} \tilde{F}_{\mu \rho}^{(i)} \tilde{F}_{\nu \sigma}^{(i)},
$$

where $\tilde{F}_{\mu \nu}^{(i)} \equiv \partial_{\mu} B_{\nu}^{(i)}-\partial_{\nu} B_{\mu}^{(i)}$.

To summarize, the Lagrangian (36) is given by

$\mathcal{L}=\sqrt{-\tilde{g}}\left\{\frac{1}{2} M_{p}^{2} \tilde{R}-\frac{1}{2} \tilde{g}^{\mu \nu} \partial_{\mu} \sigma \partial_{\nu} \sigma+\sum_{i=1}^{n}\left[-\frac{1}{4}\left(\tilde{F}_{\mu \nu}^{(i)}\right)^{2}-\frac{e_{i}^{2}}{2 n \xi_{i}} M_{p}^{2} B_{\mu}^{(i)} B^{(i) \mu}\right]\right\}$,

where the definition $\zeta^{-2}=6+\frac{1}{n} \sum_{i=1}^{n} \frac{1}{\xi_{i}}$ is used. It is then obvious that this system also exhibits the new Higgs mechanism triggered by spontaneous symmetry breakdown of scale symmetry.

\section{Radiative corrections and dilaton mass}

In this section, as an example, we wish to apply our idea discussed so far to the standard model and evaluate the quantum effects. Since the standard model is known to not be classically scale-invariant because of the presence of the 
(negative) mass term of the Higgs field, we must replace the mass term with a new scalar field. It is then natural to identify this new scalar with the $\Phi$ field which couples to a scalar curvature in a non-minimal manner.

Let us first recall that in our previous work [11] we have already considered one-loop effects of a classically scale invariant B-L model [8]. However, our finding of gravitational spontaneous symmetry breakdown of gauge symmetry as a result of spontaneous symmetry breakdown of scale symmetry is very universal in the sense that our ideas can be generalized not only to local scale symmetry as clarified in [12] but also to non-Abelian gauge groups and even a scalar field with many of components as discussed in this article. In fact, it is obvious that our ideas can be applied to any model which is scale-invariant and involves the non-minimal coupling terms between the curvature scalar and charged scalars associated with local gauge symmetries.

In our calculation, we are not ambitious enough to quantize the metric tensor field and take a fixed Minkowski background $g_{\mu \nu}=\eta_{\mu \nu}$. Moreover, we restrict ourselves to the calculation of radiative corrections between dilaton and matter fields in the weak-field approximation.

One of the motivations behind this study is to calculate the size of the mass of dilaton. As shown above, the dilaton is exactly massless at the classical level owing to scale symmetry, but it is well-known that radiative corrections violate the scale invariance thereby leading to the trace anomaly. Consequently, the dilaton becomes massive in the quantum regime. Since the dilaton is a scalar field like the Higgs particle, one might expect that there could be quadratic divergence for the self-energy diagram. On the other hand, since the dilaton is the Nambu-Goldstone boson resulting from the scale symmetry, the dilaton mass would be much lower in the such a way that the pion masses are very smaller as the pions can be understood as the Nambu-Goldstone boson coming from $S U(2)_{L} \times S U(2)_{R} \rightarrow S U(2)_{V}$ flavor symmetry breaking. As long as we know, nobody has calculated the dilaton mass in a reliable manner, so we wish to calculate the dilaton mass within the framework of the present formalism and determine which scenario, quadratic divergence and huge radiative corrections like the Higgs particle or very lower mass like the pions, is realized. Remarkably enough, it will be shown that although the dilaton mass is quadratic divergent, the cutoff scale, which is the Planck mass in the formalism at hand, is exactly cancelled by the induced coupling constant, by which the dilaton mass is kept to be around the GeV scale.

Whenever we evaluate anomalies, the key point is to adopt a suitable regularization method respecting classical symmetries existing in the action as much as possible. In this article, as a regularization method, we make use of the method of continuous space-time dimensions, for which we rewrite previous results in arbitrary $D$ dimensions [17]. Like the dimensional regularization, the divergences will appear as poles $\frac{1}{D-4}$, which are cancelled by the factor $D-4$ 
that multiplies the dilaton coupling, thereby producing a finite result leading to an effective interaction term.

\subsection{Basic formalism}

Our starting Lagrangian, which is a scale-invariant extension of the standard model coupled to the non-minimal terms, is of form

$$
\begin{aligned}
\mathcal{L} & =\sqrt{-g}\left[\left(\xi_{1} \Phi^{\dagger} \Phi+\xi_{2} H^{\dagger} H\right) R-g^{\mu \nu}\left(D_{\mu} \Phi\right)^{\dagger}\left(D_{\nu} \Phi\right)-g^{\mu \nu}\left(D_{\mu} H\right)^{\dagger}\left(D_{\nu} H\right)\right. \\
& \left.-\frac{1}{4} g^{\mu \nu} g^{\rho \sigma}\left(F_{\mu \rho}^{(1)} F_{\nu \sigma}^{(1)}+F_{\mu \rho}^{(2)} F_{\nu \sigma}^{(2)}+F_{\mu \rho}^{a} F_{\nu \sigma}^{a}\right)-V(H, \Phi)+L_{m}\right]
\end{aligned}
$$

where $L_{m}$ denotes the remaining Lagrangian part of the standard-model sector such as the Yukawa couplings and various definitions are given by the following expressions:

$$
\begin{aligned}
D_{\mu} \Phi & =\left(\partial_{\mu}-i e_{1} A_{\mu}^{(1)}\right) \Phi, \quad D_{\mu} H=\left(\partial_{\mu}-i g \tau^{a} A_{\mu}^{a}-i \frac{e_{2}}{2} A_{\mu}^{(2)}\right) H, \\
F_{\mu \nu}^{(i)} & =\partial_{\mu} A_{\nu}^{(i)}-\partial_{\nu} A_{\mu}^{(i)}, \quad F_{\mu \nu}^{a}=\partial_{\mu} A_{\nu}^{a}-\partial_{\nu} A_{\mu}^{a}+g \varepsilon^{a b c} A_{\mu}^{b} A_{\nu}^{c}, \\
V(H, \Phi) & =\lambda_{\Phi}\left(\Phi^{\dagger} \Phi\right)^{2}+\lambda_{H \Phi}\left(H^{\dagger} H\right)\left(\Phi^{\dagger} \Phi\right)+\lambda_{H}\left(H^{\dagger} H\right)^{2} .
\end{aligned}
$$

with $e_{i}(i=1,2)$ being $U(1)$ coupling constants and $g$ being an $S U(2)$ coupling constant. As in the Appendix A, in this model, we can also calculate the Noether current for scale transformation

$$
J^{\mu}=\sqrt{-g} g^{\mu \nu} \partial_{\nu}\left[\left(6 \xi_{1}+1\right) \Phi^{\dagger} \Phi+\left(6 \xi_{2}+1\right) H^{\dagger} H\right] .
$$

It turns out that this dilatation current is conserved on-shell as well.

For simplicity of presentation, we take the vanishing $S U(2)$ gauge field, $A_{\mu}^{a}=0$ since this assumption does not change the essential conclusion for our purpose.

In general $D$ space-time dimensions, as a generalization of Eq. (16), the local scale transformation is defined as

$$
\begin{aligned}
\hat{g}_{\mu \nu} & =\Omega^{2}(x) g_{\mu \nu}, \quad \hat{g}^{\mu \nu}=\Omega^{-2}(x) g^{\mu \nu}, \quad \hat{\Phi}=\Omega^{-\frac{D-2}{2}}(x) \Phi, \\
\hat{H} & =\Omega^{-\frac{D-2}{2}}(x) H, \quad \hat{A}_{\mu}^{(i)}=\Omega^{-\frac{D-4}{2}}(x) A_{\mu}^{(i)} .
\end{aligned}
$$

Under this local scale transformation (49), with the definition $f=\log \Omega$, the scalar curvature is transformed as

$$
R=\Omega^{2}\left[\hat{R}+2(D-1) \square f-(D-1)(D-2) \hat{g}^{\mu \nu} \partial_{\mu} f \partial_{\nu} f\right],
$$

for which we set $D=4$ in what follows since we do not quantize the metric tensor and therefore do not have poles from the curvature. 
In a physically more realistic situation, the scale symmetry must be broken spontaneously in the higher energy region before spontaneous symmetry breaking of the electro-weak symmetry since all quantum field theories must in principle contain the gravity from the beginning although contributions from the gravity can be usually ignored when dealing with particle physics processes.

Therefore, let us first break the scale invariance by taking the following value for the charged scalar field $\Phi$ :

$$
\Phi=\frac{1}{\sqrt{2 \xi_{1}}} \Omega^{\frac{D-2}{2}} e^{i \alpha \theta}=\frac{1}{\sqrt{2 \xi_{1}}} e^{\zeta \sigma+i \alpha \theta}
$$

where we have defined $\Omega(x)=e^{\frac{2}{D-2} \zeta \sigma}$ and $\zeta^{-2} \equiv 4 \frac{D-1}{D-2}+\frac{1}{\xi_{1}}=6+\frac{1}{\xi_{1}}$. Then, the first term in (46) takes the form

$$
\sqrt{-g} \xi_{1} \Phi^{\dagger} \Phi R=\sqrt{-\hat{g}}\left(\frac{1}{2} \hat{R}-3 \zeta^{2} \hat{g}^{\mu \nu} \partial_{\mu} \sigma \partial_{\nu} \sigma\right)
$$

Similarly, the third term in (46) is changed to the form

$$
-\sqrt{-g} g^{\mu \nu}\left(D_{\mu} \Phi\right)^{\dagger}\left(D_{\nu} \Phi\right)=-\frac{1}{2 \xi_{1}} \sqrt{-\hat{g}} \hat{g}^{\mu \nu}\left(\zeta^{2} \partial_{\mu} \sigma \partial_{\nu} \sigma+\hat{e}_{1}^{2} \hat{B}_{\mu}^{(1)} \hat{B}_{\nu}^{(1)}\right)
$$

where we have defined a new coupling constant and massive gauge field as

$$
\hat{e}_{1}=\Omega^{\frac{D-4}{2}} e_{1}, \quad \hat{B}_{\mu}^{(1)}=\hat{A}_{\mu}^{(1)}-\partial_{\mu} \theta,
$$

and chosen $\alpha=\hat{e}_{1}$ for convenience. Adding (52) and (53) together yields the expression

$$
\sqrt{-g}\left[\xi_{1} \Phi^{\dagger} \Phi R-g^{\mu \nu}\left(D_{\mu} \Phi\right)^{\dagger}\left(D_{\nu} \Phi\right)\right]=\sqrt{-\hat{g}}\left(\frac{1}{2} \hat{R}-\frac{1}{2} \hat{g}^{\mu \nu} \partial_{\mu} \sigma \partial_{\nu} \sigma-\frac{\hat{e}_{1}^{2}}{2 \xi_{1}} \hat{g}^{\mu \nu} \hat{B}_{\mu}^{(1)} \hat{B}_{\nu}^{(1)}\right)
$$

On the other hand, the Lagrangian of matter fields turns out to depend on the dilaton field $\sigma$ in a non-trivial manner in general $D$ space-time dimensions. First, the non-minimal term for $H$ field becomes

$$
\sqrt{-g} \xi_{2} H^{\dagger} H R=\sqrt{-\hat{g}} \xi_{2} \hat{H}^{\dagger} \hat{H}\left(\hat{R}+6 \zeta^{2} \square \sigma-6 \zeta^{2} \hat{g}^{\mu \nu} \partial_{\mu} \sigma \partial_{\nu} \sigma\right) .
$$

Second, the kinetic term for $H$ is cast to

$$
-\sqrt{-g} g^{\mu \nu}\left(D_{\mu} H\right)^{\dagger}\left(D_{\nu} H\right)=-\sqrt{-\hat{g}} \hat{g}^{\mu \nu}\left(\hat{D}_{\mu} \hat{H}\right)^{\dagger}\left(\hat{D}_{\nu} \hat{H}\right),
$$

where the new covariant derivative is defined as

$$
\hat{D}_{\mu} \hat{H}=\left(\partial_{\mu}+\zeta \partial_{\mu} \sigma-\frac{i}{2} \hat{e}_{2} \hat{A}_{\mu}^{(2)}\right) \hat{H}
$$


with being $\hat{e}_{2}=\Omega^{\frac{D-4}{2}} e_{2}$. Third, the electro-magnetic terms are reduced to the form

$$
-\frac{1}{4} \sqrt{-g} \sum_{i=1}^{2} g^{\mu \nu} g^{\rho \sigma} F_{\mu \rho}^{(i)} F_{\nu \sigma}^{(i)}=-\frac{1}{4} \sqrt{-\hat{g}} \sum_{i=1}^{2} \hat{g}^{\mu \nu} \hat{g}^{\rho \sigma} \hat{F}_{\mu \rho}^{(i)} \hat{F}_{\nu \sigma}^{(i)},
$$

where the new field strengths are defined $\mathrm{as}^{6}$

$$
\begin{aligned}
& \hat{F}_{\mu \nu}^{(1)}=\Omega^{2-\frac{D}{2}} F_{\mu \nu}^{(1)}=\partial_{\mu} \hat{B}_{\nu}^{(1)}+\frac{D-4}{2} \zeta \partial_{\mu} \sigma\left(\hat{B}_{\nu}^{(1)}+\partial_{\nu} \theta\right)-(\mu \leftrightarrow \nu), \\
& \hat{F}_{\mu \nu}^{(2)}=\Omega^{2-\frac{D}{2}} F_{\mu \nu}^{(2)}=\partial_{\mu} \hat{A}_{\nu}^{(2)}+\frac{D-4}{2} \zeta \partial_{\mu} \sigma \hat{A}_{\nu}^{(2)}-(\mu \leftrightarrow \nu) .
\end{aligned}
$$

Finally, the potential term can be rewritten as

$$
\begin{aligned}
\sqrt{-g} V(H, \Phi) & =\sqrt{-\hat{g}} V(\hat{H}) \\
& =\sqrt{-\hat{g}} e^{\frac{2(D-4)}{D-2}} \zeta \sigma\left[\frac{1}{4 \xi_{1}^{2}} \lambda_{\Phi} M_{p}^{4}+\frac{1}{2 \xi_{1}} \lambda_{H \Phi} M_{p}^{2}\left(\hat{H}^{\dagger} \hat{H}\right)+\lambda_{H}\left(\hat{H}^{\dagger} \hat{H} \gamma \hat{\jmath}\right]\right)
\end{aligned}
$$

To summarize, the starting Lagrangian is now of the form

$$
\begin{aligned}
\mathcal{L} & =\sqrt{-\hat{g}}\left[\frac{1}{2} \hat{R}-\frac{1}{2} \hat{g}^{\mu \nu} \partial_{\mu} \sigma \partial_{\nu} \sigma-\frac{\hat{e}_{1}^{2}}{2 \xi_{1}} \hat{g}^{\mu \nu} \hat{B}_{\mu}^{(1)} \hat{B}_{\nu}^{(1)}\right. \\
& +\xi_{2} \hat{H}^{\dagger} \hat{H}\left(\hat{R}+6 \zeta^{2} \square \sigma-6 \zeta^{2} \hat{g}^{\mu \nu} \partial_{\mu} \sigma \partial_{\nu} \sigma\right)-\hat{g}^{\mu \nu}\left(\hat{D}_{\mu} \hat{H}\right)^{\dagger}\left(\hat{D}_{\nu} \hat{H}\right) \\
& \left.-\frac{1}{4} \sum_{i=1}^{2} \hat{g}^{\mu \nu} \hat{g}^{\rho \sigma} \hat{F}_{\mu \rho}^{(i)} \hat{F}_{\nu \sigma}^{(i)}-V(\hat{H})+L_{m}\right] .
\end{aligned}
$$

Next, we are ready to deal with spontaneous symmetry breakdown of the electro-weak symmetry, which is assumed to occur at the lower energy, GeV scale, than breaking of scale symmetry. To realize the spontaneous symmetry breakdown of the electro-weak symmetry, we assume the conventional ansatz

$$
\lambda_{H \Phi}<0, \quad \lambda_{H}>0 .
$$

With the parametrization $\hat{H}^{T}=\left(0, \frac{v+h}{\sqrt{2}}\right) e^{i \varphi}$, after the spontaneous symmetry breakdown of the electro-weak symmetry, the potential term can be described as

$$
V(\hat{H})=e^{\frac{2(D-4)}{D-2} \zeta \sigma}\left[\frac{1}{2} m_{h}^{2} h^{2}+\sqrt{\frac{\lambda_{H}}{2}} m_{h} h^{3}+\frac{\lambda_{H}}{4} h^{4}\right],
$$

\footnotetext{
${ }^{6}$ The presence of the Nambu-Goldstone mode $\theta$ in $\hat{F}_{\mu \nu}^{(1)}$ merely shows that scale invariance of the theory under consideration is violated in any space-time dimension except four dimensions.
} 
where a constant in the square bracket is discarded, and the vacuum expectation value $v$ and the Higgs mass $m_{h}$ are respectively defined as

$$
v^{2}=\frac{1}{2 \xi_{1}} \frac{\left|\lambda_{H \Phi}\right|}{\lambda_{H}} M_{p}^{2}, \quad m_{h}=\sqrt{2 \lambda_{H}} v=\sqrt{\frac{\left|\lambda_{H \Phi}\right|}{\xi_{1}}} M_{p},
$$

where the Planck mass $M_{p}$ is explicitly written.

Now we wish to consider couplings between the dilaton field $\sigma$ and matter fields which vanish at the classical level $(D=4)$ but provide a finite contribution at the quantum level, interpreted as the trace anomaly. In the weak field approximation, let us extract terms linear in the dilaton $\sigma$ in $V(\hat{H})$ as

$$
e^{\frac{2(D-4)}{D-2} \zeta \sigma} \approx 1+(D-4) \zeta \sigma
$$

Then, the potential $V(\hat{H})$ is devided into two parts

$$
V(\hat{H})=V^{(0)}(\hat{H})+V^{(1)}(\hat{H})
$$

where we have defined as

$$
\begin{aligned}
V^{(0)}(\hat{H}) & =\frac{1}{2} m_{h}^{2} h^{2}+\sqrt{\frac{\lambda_{H}}{2}} m_{h} h^{3}+\frac{\lambda_{H}}{4} h^{4}, \\
V^{(1)}(\hat{H}) & =(D-4) \zeta V^{(0)}(\hat{H}) \sigma .
\end{aligned}
$$

Using the parametrization $\hat{H}^{T}=\left(0, \frac{v+h}{\sqrt{2}}\right) e^{i \varphi}$, the remaining part including the field $H$ except the Higgs potential is also rewritten, and consequently the whole Lagrangian (62) takes a little longer expression

$$
\begin{aligned}
\mathcal{L} & =\sqrt{-\hat{g}}\left\{\frac{1}{2} M_{p}^{2} \hat{R}-\frac{1}{2} \hat{g}^{\mu \nu} \partial_{\mu} \sigma \partial_{\nu} \sigma-\frac{\hat{e}_{1}^{2}}{2 \xi_{1}} M_{p}^{2} \hat{g}^{\mu \nu} \hat{B}_{\mu}^{(1)} \hat{B}_{\nu}^{(1)}\right. \\
& +\frac{1}{2} \xi_{2} v^{2} \hat{R}+\xi_{2}\left(v h+\frac{1}{2} h^{2}\right)\left(\hat{R}-6 \zeta^{2} \frac{1}{M_{p}^{2}} \hat{g}^{\mu \nu} \partial_{\mu} \sigma \partial_{\nu} \sigma\right) \\
& -3 \xi_{2} \zeta^{2} \frac{v^{2}}{M_{p}^{2}} \hat{g}^{\mu \nu} \partial_{\mu} \sigma \partial_{\nu} \sigma-6 \xi_{2} \zeta^{2} \frac{v}{M_{p}} \hat{g}^{\mu \nu} \partial_{\mu} h \partial_{\nu} \sigma+3 \xi_{2} \zeta^{2} \frac{1}{M_{p}} h^{2} \square \sigma \\
& -\hat{g}^{\mu \nu}\left[\frac{1}{2} \partial_{\mu} h \partial_{\nu} h+\zeta \frac{1}{M_{p}} \partial_{\mu} h \partial_{\nu} \sigma(v+h)+\frac{1}{2} \zeta^{2} \frac{1}{M_{p}^{2}} \partial_{\mu} \sigma \partial_{\nu} \sigma(v+h)^{2}\right. \\
& \left.\left.+\frac{\hat{e}_{2}^{2}}{8} \hat{A}_{\mu}^{(2)} \hat{A}_{\nu}^{(2)}(v+h)^{2}\right]-\frac{1}{4} \sum_{i=1}^{2} \hat{g}^{\mu \nu} \hat{g}^{\rho \sigma} \hat{F}_{\mu \rho}^{(i)} \hat{F}_{\nu \sigma}^{(i)}-V(\hat{H})+L_{m}\right\},
\end{aligned}
$$

where we have recovered the Planck mass scale $M_{p}$. As mentioned in Section 4 , given two non-minimal terms, we need to diagonalize the kinetic terms for the dilaton $\sigma$ and the Higgs field $h$ to get the canonical form. However, in the present context, the energy scale $v$ of the electro-weak symmetry breaking is 
much lower compared to that of the scale symmetry one, so it is reasonable to take the approximation

$$
\frac{v}{M_{p}} \ll 1, \quad \xi_{2} v^{2} \ll M_{p}^{2}
$$

With this approximation, the Lagrangian is rather simplified to

$$
\begin{aligned}
\mathcal{L} & =\sqrt{-\hat{g}}\left[\frac{1}{2} \hat{R}-\frac{1}{2} \hat{g}^{\mu \nu} \partial_{\mu} \sigma \partial_{\nu} \sigma-\frac{\hat{e}_{1}^{2}}{2 \xi_{1}} \hat{g}^{\mu \nu} \hat{B}_{\mu}^{(1)} \hat{B}_{\nu}^{(1)}\right. \\
& +\xi_{2}\left(v h+\frac{1}{2} h^{2}\right) \hat{R}-\frac{1}{2} \hat{g}^{\mu \nu} \partial_{\mu} h \partial_{\nu} h-\frac{\hat{e}_{2}^{2}}{8} \hat{g}^{\mu \nu} \hat{A}_{\mu}^{(2)} \hat{A}_{\nu}^{(2)}(v+h)^{2} \\
& \left.-\frac{1}{4} \sum_{i=1}^{2} \hat{g}^{\mu \nu} \hat{g}^{\rho \sigma} \hat{F}_{\mu \rho}^{(i)} \hat{F}_{\nu \sigma}^{(i)}-V(\hat{H})+L_{m}\right] .
\end{aligned}
$$

Based on this Lagrangian, we wish to calculate quantum effects, in particular, on the dilaton coupling below. Since we are interested in the low energy region, the derivative coupling of the dilaton appearing in $\hat{F}_{\mu \nu}^{(i)}$ and $\hat{D}_{\mu} \hat{H}$ will be ignored in the calculation.

\subsection{The coupling between dilaton and Higgs field}

In this subsection, we first switch off the $U(1)$ fields and calculate the coupling between the dilaton $\sigma$ and the Higgs particle $h$ and derive an effective Lagrangian at the one-loop level. The contribution from the $U(1)$ fields will be discussed in the later subsection. We will see that the $\sigma h^{n}(2 \leq n \leq 4)$ $(n+1)$-point diagrams are non-vanishing whereas the $\sigma h^{n}(n \geq 5)$ diagrams are vanishing.

First, let us consider three-point (with two Higgs $h$ and one dilaton $\sigma$ as the external particles), one-loop diagrams. Inspection of the vertices reveals that we have three types of one-loop divergent diagrams in which the Higgs field is circulating in the loop and one dilaton field, whose momentum is assumed to be vanishing, couples. Note that the divergences stemming from the Higgs one-loop diagrams provide us with poles $\frac{1}{D-4}$, which cancel the factor $D-4$ multiplying the dilaton coupling in $V^{(1)}(\hat{H})$, thereby yielding a finite contribution.

One type of one-loop divergent diagram, which we call the diagram (A1), is a tadpole type and is given by the Higgs loop to which the dilaton couples by the vertex $-(D-4) 3 ! \zeta \lambda_{H}$ in $V^{(1)}(\hat{H})$. The corresponding amplitude $\mathcal{T}_{A 1}$ is of form

$$
\mathcal{T}_{A 1}=-i(D-4) 3 ! \zeta \lambda_{H} \int \frac{d^{D} k}{(2 \pi)^{D}} \frac{1}{k^{2}+m_{h}^{2}}
$$




$$
\begin{aligned}
& =-i(D-4) 3 ! \zeta \lambda_{H} \frac{i \pi^{2}}{(2 \pi)^{4}}\left(m_{h}^{2}\right)^{\frac{D}{2}-1} \Gamma\left(1-\frac{D}{2}\right) \\
& =\frac{3}{4 \pi^{2}} \zeta \lambda_{H} m_{h}^{2},
\end{aligned}
$$

where we have used the familiar formula in the dimensional regularization which corresponds to a specific case of the general formula in Appendix B

$$
\int \frac{d^{D} k}{(2 \pi)^{D}} \frac{1}{k^{2}+m_{h}^{2}}=\frac{i \pi^{2}}{(2 \pi)^{4}}\left(m_{h}^{2}\right)^{\frac{D}{2}-1} \Gamma\left(1-\frac{D}{2}\right)
$$

and the property of the gamma function $\Gamma(m+1)=m \Gamma(m)$.

The second type of one-loop divergent diagram, which we call the diagram (A2), is given by the Higgs loop to which the dilaton couples by the vertex $-(D-4) \zeta m_{h}^{2}$ in $V^{(1)}(\hat{H})$ and with the Higgs self-coupling vertex $-3 ! \lambda_{H}$ in $V^{(0)}(\hat{H})$. The amplitude $\mathcal{T}_{A 2}$ is calculated as

$$
\begin{aligned}
\mathcal{T}_{A 2} & =i(D-4) 3 ! \zeta \lambda_{H} m_{h}^{2} \int \frac{d^{D} k}{(2 \pi)^{D}} \frac{1}{\left(k^{2}+m_{h}^{2}\right)^{2}} \\
& =i(D-4) 3 ! \zeta \lambda_{H} m_{h}^{2} \frac{i}{16 \pi^{2}} \Gamma\left(2-\frac{D}{2}\right) \\
& =\frac{3}{4 \pi^{2}} \zeta \lambda_{H} m_{h}^{2},
\end{aligned}
$$

where we have used the equation

$$
\begin{aligned}
\int \frac{d^{D} k}{(2 \pi)^{D}} \frac{1}{\left(k^{2}+m_{h}^{2}\right)^{2}} & =-\frac{\partial}{\partial m_{h}^{2}} \int \frac{d^{D} k}{(2 \pi)^{D}} \frac{1}{k^{2}+m_{h}^{2}} \\
& =\frac{i \pi^{2}}{(2 \pi)^{4}}\left(m_{h}^{2}\right)^{\frac{D}{2}-2}\left(1-\frac{D}{2}\right) \Gamma\left(1-\frac{D}{2}\right) \\
& =\frac{i}{16 \pi^{2}} \Gamma\left(2-\frac{D}{2}\right) .
\end{aligned}
$$

The final type of one-loop diagram, which we call the diagram (A3), is a little more involved and given by the Higgs loop to which the dilaton couples by the vertex $-(D-4) 3 ! \zeta \sqrt{\frac{\lambda_{H}}{2}} m_{h}$ in $V^{(1)}(\hat{H})$ and with the Higgs self-coupling vertex $-3 ! \sqrt{\frac{\lambda_{H}}{2}} m_{h}$ in $V^{(0)}(\hat{H})$. The amplitude $\mathcal{T}_{A 3}$ reads

$$
\begin{aligned}
\mathcal{T}_{A 3} & =2 i(D-4) \zeta\left(-3 ! \sqrt{\frac{\lambda_{H}}{2}} m_{h}\right)^{2} \int \frac{d^{D} k}{(2 \pi)^{D}} \frac{1}{\left(k^{2}+m_{h}^{2}\right)\left[(k+q)^{2}+m_{h}^{2}\right]} \\
& =36 i \zeta \lambda_{H} m_{h}^{2}(D-4) \frac{i}{16 \pi^{2}} \Gamma\left(2-\frac{D}{2}\right) \\
& =\frac{9}{2 \pi^{2}} \zeta \lambda_{H} m_{h}^{2},
\end{aligned}
$$


where $q$ is the external momentum of the Higgs field. In order to reach the final result in Eq. (76), we have evaluated the integral as follows:

$$
\begin{aligned}
I & =\int d^{D} k \frac{1}{\left(k^{2}+m_{h}^{2}\right)\left[(k+q)^{2}+m_{h}^{2}\right]} \\
& =\int_{0}^{1} d x \int d^{D} k \frac{1}{\left[\left(k^{2}+m_{h}^{2}\right)(1-x)+\left((k+q)^{2}+m_{h}^{2}\right) x\right]^{2}} \\
& =\int_{0}^{1} d x \int d^{D} k \frac{1}{\left[(k+x q)^{2}+m_{h}^{2}+x(1-x) q^{2}\right]^{2}} \\
& =\int_{0}^{1} d x \int d^{D} k \frac{1}{\left[k^{2}+m_{h}^{2}+x(1-x) q^{2}\right]^{2}} \\
& =\int_{0}^{1} d x i \pi^{2} \Gamma\left(2-\frac{D}{2}\right)\left(m_{h}^{2}\right)^{\frac{D}{2}-2}\left(1-x+x^{2}\right)^{\frac{D}{2}-2} \\
& =i \pi^{2} \Gamma\left(2-\frac{D}{2}\right) .
\end{aligned}
$$

Here at the second equality, we have used the Feynman parameter formula (145) and at the fourth equality, we have shifted the momentum $k+x q \rightarrow k$, which is allowed since the integral is now finite owing to the regularization, and at the fifth equality we have used the on-mass-shell condition $q^{2}=-m_{h}^{2}$ and Eq. (75).

Thus, adding three types of contributions, we have

$$
\mathcal{T}_{A}=\mathcal{T}_{A 1}+\mathcal{T}_{A 2}+\mathcal{T}_{A 3}=\frac{6}{\pi^{2}} \zeta \lambda_{H} m_{h}^{2}
$$

From this result, we can construct an effective Lagrangian at the one-loop level

$$
L_{\sigma h^{2}}=-\frac{3}{\pi^{2}} \zeta \lambda_{H} \frac{m_{h}^{2}}{M_{p}} \sigma h^{2}
$$

where we have explicitly written down the Planck mass dependence in such a way that we can recognize dimensions clearly.

Next, let us take account of four-point (with three Higgs and one dilaton as the external particles), one-loop diagrams. In this case, inspection of the vertices reveals again that there are two types of one-loop divergent diagrams where the Higgs field is circulating in the loop. One type of one-loop divergent diagram, which we call the diagram (B1), is given by the Higgs loop to which the dilaton couples by the vertex $-(D-4) 3 ! \zeta \lambda_{H}$ in $V^{(1)}(\hat{H})$ and with the Higgs self-coupling $-3 ! \sqrt{\frac{\lambda_{H}}{2}} m_{h}$ in $V^{(0)}(\hat{H})$. The corresponding amplitude $\mathcal{T}_{B 1}$ reads

$$
\mathcal{T}_{B 1}=i(D-4) 3 ! \zeta \lambda_{H} \sqrt{\frac{\lambda_{H}}{2}} m_{h} 3 ! \int \frac{d^{D} k}{(2 \pi)^{D}} \frac{1}{\left(k^{2}+m_{h}^{2}\right)\left[(k+q)^{2}+m_{h}^{2}\right]}
$$




$$
\begin{aligned}
& =36 i \zeta \lambda_{H} \sqrt{\frac{\lambda_{H}}{2}} m_{h}(D-4) \frac{i}{16 \pi^{2}} \Gamma\left(2-\frac{D}{2}\right) \\
& =\frac{9}{2 \sqrt{2} \pi^{2}} \zeta \lambda_{H} \sqrt{\lambda_{H}} m_{h},
\end{aligned}
$$

where we have used Eq. (77).

The other type of one-loop divergent diagram, which is called the diagram (B2), is given by the Higgs loop to which the dilaton couples by the vertex $-(D-4) 3 ! \zeta \sqrt{\frac{\lambda_{H}}{2}} m_{h}$ in $V^{(1)}(\hat{H})$ and with the Higgs self-coupling $-3 ! \lambda_{H}$ in $V^{(0)}(\hat{H})$. The amplitude $\mathcal{T}_{B 2}$ takes the form

$$
\begin{aligned}
\mathcal{T}_{B 2} & =i(D-4) 3 ! \zeta \sqrt{\frac{\lambda_{H}}{2}} m_{h} 3 ! \lambda_{H} \int \frac{d^{D} k}{(2 \pi)^{D}} \frac{1}{\left(k^{2}+m_{h}^{2}\right)\left[(k+q)^{2}+m_{h}^{2}\right]} \\
& =\frac{9}{2 \sqrt{2} \pi^{2}} \zeta \lambda_{H} \sqrt{\lambda_{H}} m_{h},
\end{aligned}
$$

Putting the two types of contributions together, we obtain

$$
\mathcal{T}_{B}=\mathcal{T}_{B 1}+\mathcal{T}_{B 2}=\frac{9}{\sqrt{2} \pi^{2}} \zeta \lambda_{H} \sqrt{\lambda_{H}} m_{h}
$$

This result gives rise to an effective Lagrangian

$$
L_{\sigma h^{3}}=-\frac{3}{2 \sqrt{2} \pi^{2}} \zeta \lambda_{H} \sqrt{\lambda_{H}} \frac{m_{h}}{M_{p}} \sigma h^{3},
$$

where we have recovered the Planck mass again.

Now we turn our attention to five-point (with four Higgs and one dilaton as the external particles), one-loop diagrams. In this case, we find that there is only one type of one-loop divergent diagram where the Higgs field is circulating in the loop. This type of one-loop divergent diagram, which we call the diagram (C), is given by the Higgs loop to which the dilaton couples by the vertex $-(D-4) 3 ! \zeta \lambda_{H}$ in $V^{(1)}(\hat{H})$ and with the Higgs self-coupling $-3 ! \lambda_{H}$ in $V^{(0)}(\hat{H})$. The corresponding amplitude $\mathcal{T}_{C}$ reads

$$
\begin{aligned}
\mathcal{T}_{C} & =2 i(D-4) 3 ! \zeta \lambda_{H} 3 ! \lambda_{H} \int \frac{d^{D} k}{(2 \pi)^{D}} \frac{1}{\left(k^{2}+m_{h}^{2}\right)\left[(k+p+q)^{2}+m_{h}^{2}\right]} \\
& =\frac{1}{\pi^{2}} \zeta \lambda_{H}^{2},
\end{aligned}
$$

where $p$ and $q$ are external momenta of the two Higgs fields. This quantum effect gives us an effective Lagrangian

$$
L_{\sigma h^{4}}=-\frac{1}{24 \pi^{2}} \zeta \lambda_{H}^{2} \frac{1}{M_{p}} \sigma h^{4} .
$$


Finally, it is straightforward to evaluate $(n+1)$-point (with $n \geq 5$ Higgs and one dilaton as external particles), one-loop diagrams in a similar manner. It turns out that these higher-point, one-loop diagrams do not yield any divergence, thereby leading to the vanishing effective Lagrangian. Moreover, we find that there are no divergences for the $\sigma^{n} h^{m}(n \geq 2)$-type of amplitudes at the one-loop level.

After all, we have a total effective Lagrangian at the one-loop level

$$
L^{1-\text { loop }}=\left[-\frac{3}{\pi^{2}} \zeta \lambda_{H} \frac{m_{h}^{2}}{M_{p}} h^{2}-\frac{3}{2 \sqrt{2} \pi^{2}} \zeta \lambda_{H} \sqrt{\lambda_{H}} \frac{m_{h}}{M_{p}} h^{3}-\frac{1}{24 \pi^{2}} \zeta \lambda_{H}^{2} \frac{1}{M_{p}} h^{4}\right] \sigma .
$$

Note that this effective Lagrangian has the similar form to the potential as $V^{(1)}(\hat{H})$ but each coefficient is suppressed by the Planck mass, which means that effects of radiative corrections are very tiny in the low energy region.

\subsection{Yukawa coupling}

In the standard model, the fermion masses arise from the Yukawa coupling between the fermions and the Higgs field. It is therefore of interest to evaluate radiative corrections of the Yukawa coupling in the present model.

It is easy to see that there are no radiative corrections to the coupling between the dilaton and the fermions at the one-loop level, but it turns out that the one-loop induced vertex produces radiative corrections to this coupling, so we are willing to calcuclate this quantum effect in this subsection.

Before delving into the calculation, let us go back to the basics of the Yukawa coupling. The Yukawa coupling between the fermions and the Higgs field is generically given by the following Lagrangian

$$
\mathcal{L}_{H \bar{\psi} \psi}=-\sqrt{-g} g_{Y} \bar{\psi}_{L} H \psi_{R}
$$

where $g_{Y}$ is the Yukawa coupling constant, $\psi_{L}$ and $\psi_{R}$ are respectively a lefthanded, $S U(2)$-doublet spinor and a right-handed singlet spinor.

To move the Jordan frame to the Einstein frame, we use the local scale transformation (49) and its fermionic one

$$
\hat{\psi}_{L}=\Omega^{-\frac{D+2}{4}}(x) \psi_{L}, \quad \hat{\psi}_{R}=\Omega^{-\frac{D+2}{4}}(x) \psi_{R} .
$$

Under this local scale transformation, the Lagrangian (87) takes the same form

$$
\mathcal{L}_{H \bar{\psi} \psi}=-\sqrt{-\hat{g}} g_{Y} \overline{\hat{\psi}}_{L} \hat{H} \hat{\psi}_{R}
$$

With the following definitions of spinors and the unitary gauge for the Higgs field $\hat{H}$,

$$
\hat{\psi}_{L}^{T}=(\hat{\chi}, \hat{\psi}), \quad \hat{\psi}_{R}=\hat{\psi}, \quad \hat{H}^{T}=\left(0, \frac{v+h(x)}{\sqrt{2}}\right)
$$


the Lagrangian is reduced to

$$
\mathcal{L}_{H \bar{\psi} \psi}=-\sqrt{-\hat{g}}\left(M_{\psi} \overline{\hat{\psi}} \hat{\psi}+\frac{g_{Y}}{\sqrt{2}} \overline{\hat{\psi}} \hat{\psi} h\right)
$$

where we have defined $M_{\psi}=\frac{g_{Y}}{\sqrt{2}} v$.

We are now in a position to calculate the one-loop amplitude where two fermions and one dilaton appear as the external particles. In this case, there is no divergent diagram but we have a finite diagram where the fermion and the Higgs field propagate in the loop, which we call the diagram (D). In this diagram, the dilaton couples to the Higgs by the vertex $-\frac{6}{\pi^{2}} \zeta \lambda_{H} \frac{m_{h}^{2}}{M_{p}}$ in (86), which is a one-loop effect ${ }^{7}$, and two fermions couple to the Higgs by the vertex $-\frac{g_{Y}}{\sqrt{2}}$ in (91). Thus, this diagram is essentially a two-loop effect. The correponding amplitude is given by

$$
\begin{aligned}
\mathcal{T}_{D} & =-i \frac{6}{\pi^{2}} \zeta \lambda_{H} \frac{m_{h}^{2}}{M_{p}}\left(\frac{g_{Y}}{\sqrt{2}}\right)^{2} \int \frac{d^{D} k}{(2 \pi)^{D}} \frac{\not 1-\not k-M_{\psi}}{\left(k^{2}+m_{h}^{2}\right)^{2}\left[(q-k)^{2}+M_{\psi}^{2}\right]} \\
& =\frac{3}{16 \pi^{4}} \zeta \lambda_{H} g_{Y}^{2} \frac{m_{h}^{2}}{M_{\psi} M_{p}} f\left(\frac{M_{\psi}}{m_{h}}\right),
\end{aligned}
$$

where $q$ is the external momentum of the fermion field, which satisfies the on-mass-shell condition $q^{2}=-M_{\psi}^{2}$ and the function $f(x)$ is defined as

$$
f(x)=\log x+\frac{1-2 x^{2}}{\sqrt{1-4 x^{2}}} \log \left[\frac{1}{2 x}\left(1+\sqrt{1-4 x^{2}}\right)\right] .
$$

To obtain the result in Eq. (92), we have calculated the integral as follows:

$$
\begin{aligned}
J & =\int d^{D} k \frac{\not 1-\not k-M_{\psi}}{\left(k^{2}+m_{h}^{2}\right)^{2}\left[(q-k)^{2}+M_{\psi}^{2}\right]} \\
& =\int_{0}^{1} d x \int d^{D} k \frac{2(1-x)\left(\not 1-\not k-M_{\psi}\right)}{\left[\left(k^{2}+m_{h}^{2}\right)(1-x)+\left((q-k)^{2}+M_{\psi}^{2}\right) x\right]^{3}} \\
& =\int_{0}^{1} d x \int d^{D} k \frac{2(1-x)\left(\not 1-\not k-M_{\psi}\right)}{\left[(k-x q)^{2}+M_{\psi}^{2} x^{2}-m_{h}^{2} x+m_{h}^{2}\right]^{3}} \\
& =\int_{0}^{1} d x \int d^{D} k \frac{2(1-x)\left[(1-x) \not 1-\not k-M_{\psi}\right)}{\left[k^{2}+M_{\psi}^{2} x^{2}-m_{h}^{2} x+m_{h}^{2}\right]^{3}} \\
& =-2 M_{\psi} \int_{0}^{1} d x \int d^{D} k \frac{1-x}{\left[k^{2}+M_{\psi}^{2} x^{2}-m_{h}^{2} x+m_{h}^{2}\right]^{3}} \\
& =\frac{i \pi^{2}}{M_{\psi}} f\left(\frac{M_{\psi}}{m_{h}}\right) .
\end{aligned}
$$

\footnotetext{
${ }^{7}$ As will seen later, we also have the similar contribution from the $U(1)$ gauge sector at the one-loop level, but we will now neglect it since the contribution from the gauge sector is smaller than that from (86).
} 
Here at the second equality, we have used the Feynman parameter formula (146). At the fourth equality, we have shifted the momentum $k-x q \rightarrow k$, and used that $\int d^{D} k k^{\mu} F\left(k^{2}\right)=0$ for a general function $F$ in addition to $q^{\mu} \approx 0$ at the low energy. Furthermore, at the final equality, we have made use of the integral formula

$$
\int d^{D} k \frac{1}{\left(k^{2}+\Delta\right)^{3}}=\frac{i \pi^{2}}{2 \Delta}
$$

which is a specific case of a general formula (141).

From the above result, an effective Lagrangian for the interaction between the dilaton and fermions can be derived to

$$
L_{\sigma \bar{\psi} \psi}=-g_{\sigma} \overline{\hat{\psi}} \hat{\psi} \sigma
$$

where the effective coupling $g_{\sigma}$ is defined by the absolute value of $\mathcal{T}_{D}$, i.e., $g_{\sigma}=\left|\mathcal{T}_{D}\right|$.

\subsection{Dilaton mass}

As seen in the Lagrangian (71), the dilaton is exactly massless at the classical level since it is the Nambu-Goldstone boson stemming from spontaneous symmetry breakdown of scale symmetry. However, it is well-known that the scale symmetry is violated by the trace anomaly at the quantum-mechanical level, and as a result, the dilaton becomes massive.

It is very interesting to evaluate the size of the dilaton mass within the present formalism. It is in general expected that if any, the Nambu-Goldstone boson would not be so heavy as in the pions. Of course, the size of the dilaton mass would be closely related to an energy scale where the scale symmetry is broken spontaneously. On the other hand, because the dilaton is a representative example of scalar particle as well as the Higg particle, it is of interest to investigate if the dilaton would receive the quadratic divergence like the Higgs particle or not.

It turns out that at the one-loop effect, there is no quantum correction for the self-energy of the dilaton and it is at the two-loop effect that radiative corrections appear for it in the formalism at hand. Actually, we have a oneloop divergent diagram for the self-energy of the dilaton, which we call the diagram (E), where two external dilatons couple to the Higgs loop by the vertex $-(D-4) \zeta m_{h}^{2}$ in $V^{(1)}(\hat{H})$ and the vertex $-\frac{6}{\pi^{2}} \zeta \lambda_{H} \frac{m_{h}^{2}}{M_{p}}$ in (86) which is already a one-loop effect. Therefore, this one-loop diagram is essentially a two-loop contribution. The amplitude takes the form

$$
\mathcal{T}_{E}=2 i(D-4) \zeta m_{h}^{2} \frac{6}{\pi^{2}} \zeta \lambda_{H} \frac{m_{h}^{2}}{M_{p}} \int \frac{d^{D} k}{(2 \pi)^{D}} \frac{1}{\left(k^{2}+m_{h}^{2}\right)^{2}}
$$




$$
=\frac{3}{2 \pi^{4}} \zeta^{2} \lambda_{H} \frac{m_{h}^{4}}{M_{p}} .
$$

This amplitude directly gives rise to an effective action for the mass term of the dilaton

$$
L_{\sigma^{2}}^{(2)}=-\frac{3}{4 \pi^{4}} \zeta^{2} \lambda_{H} \frac{m_{h}^{4}}{M_{p}^{2}} \sigma^{2} \equiv-\frac{1}{2} m_{\sigma}^{2} \sigma^{2},
$$

where we have defined the induced dilaton mass $m_{\sigma}$ as

$$
m_{\sigma}^{2}=\frac{3}{2 \pi^{4}} \zeta^{2} \lambda_{H} \frac{m_{h}^{4}}{M_{p}^{2}} .
$$

As expected, it has turned out that the dilaton, which is massless classically, becomes massive because of radiative corrections.

From the result (99), one might be tempted to conclude that the dilaton mass induced by radiative corrections is very small since the size of the mass is suppressed by the Planck mass and $\zeta \approx \lambda_{H} \approx \mathcal{O}(1)$. But the story has not ended yet because we have to take the quadratic divergence, which is the root of the hiearchy problem in case of the Higgs particle, into consideration. Since there is no interaction vertex $\sigma^{2} h^{2}$ in the present formalism, the most severe quadratic divergence appears when the fermion is circulating in the loop via the vertex in the Lagrangian (96). The amplitude $\mathcal{T}_{F}$, which is essentially a five-loop effect, is certainly quadratically divergent by power counting

$$
\mathcal{T}_{F}=i g_{\sigma}^{2} \int \frac{d^{D} k}{(2 \pi)^{D}} \frac{1}{\left(\not k+M_{\psi}\right)^{2}} \approx i g_{\sigma}^{2} \int \frac{d^{D} k}{(2 \pi)^{D}} \frac{1}{k^{2}} \approx-g_{\sigma}^{2} \Lambda^{2}
$$

where $\Lambda$ is the ultra-violet cutoff. Then, with the reasonable choice $\Lambda=M_{p}$, the mass of the dilaton is approximately given by

$$
m_{\sigma} \approx g_{\sigma} \Lambda=\frac{3}{16 \pi^{4}} \zeta \lambda_{H} g_{Y}^{2} \frac{m_{h}^{2}}{M_{\psi}}\left|f\left(\frac{M_{\psi}}{m_{h}}\right)\right|
$$

which is around the GeV scale since $\left|f\left(\frac{M_{\psi}}{m_{h}}\right)\right| \approx 1$ for $m_{h} \approx M_{\psi}$, which holds approximately for $\psi=$ top-quark. (Here it is reasonable to take $\zeta \approx \lambda_{H} \approx$ $g_{Y} \approx \mathcal{O}(1)$ at the low energy.) Note that the factor $\frac{1}{M_{p}}$ in $g_{\sigma}$ is cancelled by the cutoff $M_{p}$. It is remarkable that the quadratic divergence, which leads to the burdensome hierarchy problem in case of the Higgs particle, gives the GeV scale mass to the dilaton! At first sight, it appears that the GeV scale mass of the dilaton is against the results of the LHC owing to null results in searches for new scalar particles except the Higgs particle below a few $\mathrm{TeV}$ scale. However, as seen in the relation $g_{\sigma}=\left|\mathcal{T}_{D}\right|$ and Eq. (92), the coupling between the dilaton and the Higgs particle is so tiny that it is extremely difficult to detect the dilaton in the LHC. 


\subsection{Contributions from gauge fields}

In the previous subsections, we have switched off the $U(1)$ gauge fields. In this final subsection, we switch on the $U(1)$ gauge fields, and wish to calculate the coupling between the dilaton and the Higgs particle by using propagators and vertices from the sector of the gauge fields in the Lagrangian (71). The result is very simple and illuminating in the sense that we can obtain the similar form of the effective Lagrangian to (86) at the one-loop level, but the coefficient of each term is multiplied by the square of the "fine structure constant".

For convenience, let us pick up part of the Lagrangian (71) which contains the gauge fields

$$
\mathcal{L}_{E M}=\sqrt{-\hat{g}}\left[-\frac{1}{4} \sum_{i=1}^{2} \hat{g}^{\mu \nu} \hat{g}^{\rho \sigma} \hat{F}_{\mu \rho}^{(i)} \hat{F}_{\nu \sigma}^{(i)}-\frac{\hat{e}_{1}^{2}}{2 \xi_{1}} \hat{g}^{\mu \nu} \hat{B}_{\mu}^{(1)} \hat{B}_{\nu}^{(1)}-\frac{\hat{e}_{2}^{2}}{8} \hat{g}^{\mu \nu} \hat{A}_{\mu}^{(2)} \hat{A}_{\nu}^{(2)}(v+h)^{2}\right](102
$$

With the following definitions of the mass of the gauge fields

$$
\hat{m}_{A}^{2}=\frac{1}{4} \hat{e}_{2}^{2} v^{2}, \quad \hat{m}_{B}^{2}=\frac{\hat{e}_{1}^{2}}{\xi_{1}}
$$

the Lagrangian (102) can be rewritten as

$\mathcal{L}_{E M}=\sqrt{-\hat{g}}\left[-\frac{1}{4} \sum_{i=1}^{2}\left(\hat{F}_{\mu \nu}^{(i)}\right)^{2}-\frac{\hat{m}_{B}^{2}}{2}\left(\hat{B}_{\mu}^{(1)}\right)^{2}-\frac{\hat{m}_{A}^{2}}{2}\left(\hat{A}_{\mu}^{(2)}\right)^{2}-\hat{m}_{A}^{2}\left(\frac{1}{v} h+\frac{1}{2 v^{2}} h^{2}\right)\left(\hat{A}_{\mu}^{(2)}\right)^{2}\right](104)$

Because of the relations $\hat{e}_{i}=\Omega^{\frac{D-4}{2}} e_{i}=e^{\frac{D-4}{D-2} \zeta \sigma} e_{i}(i=1,2)$, we have

$$
\hat{m}_{A}^{2} \approx m_{A}^{2}[1+(D-4) \zeta \sigma], \quad \hat{m}_{B}^{2} \approx m_{B}^{2}[1+(D-4) \zeta \sigma],
$$

where $m_{A}^{2}, m_{B}^{2}$ are defined as in (103) but without the hat on $e_{i}$. Thus, in the sector of the gauge fields, we have six different vertices $\sigma-B^{2}, \sigma-A^{2}, h-$ $A^{2}, h^{2}-A^{2}, \sigma-h-A^{2}, \sigma-h^{2}-A^{2}$, and two propagators of massive gauge fields $\hat{A}_{\mu}^{(2)}, \hat{B}_{\mu}^{(1)}$ for which we take the Feynman gauge.

Now, on the basis of these vertices and propagators, we would like to consider $(n+1)$-point (with $n$ Higgs particles and one dilaton as external particles), one-loop diagrams. For $n=2$, we have two types of one-loop divergent diagrams in which the gauge field $A_{\mu}^{(2)}$ is circulating in the loop. One type of the diagram, which we call the diagram (G1), is a tadpole type in which the dilaton couples to the loop composed of the gauge field $A_{\mu}^{(2)}$ by the vertex $-2 \frac{m_{A}^{2}}{v^{2}}(D-4) \zeta \eta_{\mu \nu}=-\frac{1}{2} e_{2}^{2}(D-4) \zeta \eta_{\mu \nu}$. The corresponding amplitude $\mathcal{T}_{G 1}$ is given by

$$
\begin{aligned}
\mathcal{T}_{G 1} & =-2 i e_{2}^{2}(D-4) \zeta \int \frac{d^{D} k}{(2 \pi)^{D}} \frac{1}{k^{2}+m_{A}^{2}} \\
& =\frac{1}{4 \pi^{2}} e_{2}^{2} \zeta m_{A}^{2} \\
& =\alpha_{2}^{2} \zeta v^{2},
\end{aligned}
$$


where we have introduced the "fine structure constant" $\alpha_{2}=\frac{e_{2}^{2}}{4 \pi}$.

The other type of one-loop diveregent diagram which we call the diagram (G2), is the self-energy type of the dilaton where the dilaton couples to the loop by the vertex $-\frac{1}{4} e_{2}^{2} v(D-4) \zeta \eta_{\mu \nu}$ and with the gauge-Higgs coupling vertex $-\frac{1}{4} e_{2}^{2} v \eta_{\mu \nu}$. The amplitude $\mathcal{T}_{G 2}$ is given by

$$
\begin{aligned}
\mathcal{T}_{G 2} & =i \frac{1}{2}(D-4) \zeta\left(e_{2}^{2} v\right)^{2} \int \frac{d^{D} k}{(2 \pi)^{D}} \frac{1}{\left(k^{2}+m_{A}^{2}\right)\left[(k+q)^{2}+m_{A}^{2}\right]} \\
& =\alpha_{2}^{2} \zeta v^{2}
\end{aligned}
$$

where $q$ is the external momentum of the Higgs particle.

Hence, adding the two results, we have

$$
\mathcal{T}_{G}=\mathcal{T}_{G 1}+\mathcal{T}_{G 2}=2 \alpha_{2}^{2} \zeta v^{2}
$$

from which, we obtain an effective Lagrangian

$$
L_{\sigma h^{2}}=-\alpha_{2}^{2} \zeta \frac{v^{2}}{M_{p}} \sigma h^{2} .
$$

Next, let us move to the evaluation of 4-point (with 3 Higgs particles and one dilaton as external particles), one-loop diagrams. In this case, there are two kinds of divergent diagrams. The one diagram, which we call the diagram (H1), has the $h-A^{2}$ vertex with the coefficient $-\frac{1}{4} e_{2}^{2} v \eta_{\mu \nu}$ and $\sigma-h^{2}-A^{2}$ vertex with the coefficient $-\frac{1}{2} e_{2}^{2}(D-4) \zeta \eta_{\mu \nu}$, so the amplitude takes the form

$$
\begin{aligned}
\mathcal{T}_{H 1} & =i \frac{1}{2}(D-4) \zeta e_{2}^{4} v \int \frac{d^{D} k}{(2 \pi)^{D}} \frac{1}{\left(k^{2}+m_{A}^{2}\right)\left[(k+q)^{2}+m_{A}^{2}\right]} \\
& =\alpha_{2}^{2} \zeta v,
\end{aligned}
$$

where $q$ is the momentum carried by the external Higgs particle coupled to the loop via the vertex $h-A^{2}$.

The other divergent diagram, which is called (H2), has the vertex $h^{2}-A^{2}$ with the coefficient $-\frac{1}{2} e_{2}^{2} \eta_{\mu \nu}$ and the vertex $\sigma-h-A^{2}$ with the coefficient $-\frac{1}{4} e_{2}^{2} v(D-4) \zeta \eta_{\mu \nu}$. It turns out the corresponding amplitude is the same as $\mathcal{T}_{H 1}$, so we have

$$
\mathcal{T}_{H 2}=\alpha_{2}^{2} \zeta v .
$$

Putting Eqs. (110) and (111) together, we obtain the result

$$
\mathcal{T}_{H}=\mathcal{T}_{H 1}+\mathcal{T}_{H 2}=2 \alpha_{2}^{4} \zeta v
$$

from which an effective Lagrangian becomes

$$
L_{\sigma h^{3}}=-\frac{1}{3} \alpha_{2}^{2} \zeta \frac{v}{M_{p}} \sigma h^{3} .
$$


Finally, as 5-point (with 4 Higgs particles and one dilaton as external particles), one-loop divergent diagrams, there is only one diagram constructed out of the vertex $h^{2}-A^{2}$ and the vertex $\sigma-h^{2}-A^{2}$. The amplitude $\mathcal{T}_{G 3}$ is of form

$$
\begin{aligned}
\mathcal{T}_{I} & =2 i(D-4) \zeta e_{2}^{4} \int \frac{d^{D} k}{(2 \pi)^{D}} \frac{1}{\left(k^{2}+m_{A}^{2}\right)\left[(k+p-q)^{2}+m_{A}^{2}\right]} \\
& =4 \alpha_{2}^{2} \zeta
\end{aligned}
$$

from which an effective Lagrangian can be derived to

$$
L_{\sigma h^{4}}=-\frac{1}{6} \alpha_{2}^{2} \zeta \frac{1}{M_{p}} \sigma h^{4} .
$$

Incidentally, it is easy to check that there are no divergent, one-loop diagrams for the case of $(n+1)$-point (with $n \geq 5$ Higgs and one dilaton as external particles) as before.

Putting these results together, we have the following effective Lagrangian from the sector of the gauge fields:

$$
\begin{aligned}
L_{E M}^{1-\text { loop }} & =\alpha_{2}^{2}\left[-\zeta \frac{v^{2}}{M_{p}} h^{2}-\frac{1}{3} \zeta \frac{v}{M_{p}} h^{3}-\frac{1}{6} \zeta \frac{1}{M_{p}} h^{4}\right] \sigma \\
& =\left(\frac{\alpha_{2}}{\lambda_{H}}\right)^{2}\left[-\frac{1}{2} \zeta \lambda_{H} \frac{m_{h}^{2}}{M_{p}} h^{2}-\frac{1}{3 \sqrt{2}} \zeta \lambda_{H} \sqrt{\lambda_{H}} \frac{m_{h}}{M_{p}} h^{3}-\frac{1}{6} \zeta \lambda_{H}^{2} \frac{1}{M_{p}} h^{4}(1 \alpha 6)\right.
\end{aligned}
$$

where we have used the relation $v=\frac{m_{h}}{\sqrt{2 \lambda_{H}}}$ in (65). The last equality shows that the Lagrangian (86) is more dominant than the Lagrangian (116) because of $\lambda_{H} \gg \alpha_{2}$. Thus, the results about the dilaton mass obtained in the previous subsection in essence remain unchanged even if the contribution from the gauge fields is taken into consideration.

\section{Conclusion}

In this article, we have investigated a Higgs mechanism in scale-invariant theories of gravitation in detail. After reviewing this new Higgs mechanism found in our previous articles $[11,12]$ in terms of the simplest model, we have extended the Higgs mechanism to non-Abelian gauge groups and a scalar field with many components. Since we have already considered the Higgs mechanism in a locally scale-invariant theory of gravitation, i.e., conformal gravity, the validity of this mechanism in the scale-invariant gravitational theories is very universal and therefore would have some phenomenological applications in future.

Moreover, we have spelled out quantum effects of a scale-invariant extension of the standard model in a flat Minkowski background, and examined the 
coupling between the dilaton and the Higgs particle. An intriguing observation done in our analysis is that although the mass of the dilaton is exactly zero at the classical level owing to the Nambu-Goldstone theorem, it becomes non-zero and takes a finite size around the $\mathrm{GeV}$ scale because of radiative corrections. It is worthwhile to mention that we have succeeded in deriving the size of the dilaton mass deductively by starting with a fundamental theory and without any specific assumption. As long as we know, the dilaton mass has not thus far been obtained in such a priori manner, so we think our derivation of the dilaton mass to be very interesting. As mentioned in the article, the $\mathrm{GeV}$ scale mass of the dilaton is consistent with the recent null results of new scalar particles except the Higgs particle in the LHC since the coupling constant of the dilaton is too small to detect the dilaton in the LHC. However, the dilaton with the $\mathrm{GeV}$ scale mass would have some implication in cosmology, e.g., the dilaton could become one of candidates of dark matter if it is somehow stable because of some unknown mechanism.

Our consideration in this article is confined to the quantum analysis in a fixed Minkowski background. In other words, quantum effects coming from the gravity are completely ignored because of non-renormalizability of quantum gravity. Since quantum gravity effects are of course not so dominant as quantum effects from matter fields in the low energy region, it is physically reasonable to neglect quantum effects of the gravity as the first approximation of the calculation. Nevertheless, it is of interest to take into consideration the quantum effects from the gravity. In the future, we wish to study the quantum effects from the gravitational sector in the present formalism.

Another interesting study for an application of our finding is the Higgs inflation [18]. We wish to return this problem as well in near future.

\section{Acknowledgements}

This work is supported in part by the Grant-in-Aid for Scientific Research (C) Nos. 22540287 and 25400262 from the Japan Ministry of Education, Culture, Sports, Science and Technology.

\section{A Derivation of current for scale transforma- tion}

In Appendix A, we will present a derivation of the dilatation current (4) via the Noether theorem. It is easy to show that the Lagrangian (1) is invariant under the scale transformation (3) without surface terms. Therefore, the expression of the Noether current is of form

$$
\Lambda J^{\mu}=\sum \frac{\partial \mathcal{L}}{\partial \partial_{\mu} \phi} \delta \phi,
$$


where $\phi=\left\{g_{\mu \nu}, \Phi, \Phi^{\dagger}\right\}$. Under the scale transformation (3) with a global parameter $\Omega=e^{\Lambda} \approx 1+\Lambda(|\Lambda| \ll 1)$, the current reads

$$
J^{\mu}=\frac{\partial \mathcal{L}}{\partial \partial_{\mu} g_{\rho \sigma}} 2 g_{\rho \sigma}-\frac{\partial \mathcal{L}}{\partial \partial_{\mu} \Phi} \Phi-\frac{\partial \mathcal{L}}{\partial \partial_{\mu} \Phi^{\dagger}} \Phi^{\dagger}
$$

so we have to calculate three objects $\frac{\partial \mathcal{L}}{\partial \partial_{\mu} g_{\rho \sigma}}, \frac{\partial \mathcal{L}}{\partial \partial_{\mu} \Phi}, \frac{\partial \mathcal{L}}{\partial \partial_{\mu} \Phi^{\dagger}}$ to obtain the expression of the dilatation current $J^{\mu}$. In particular, calculating the first object $\frac{\partial \mathcal{L}}{\partial \partial_{\mu} g_{\rho \sigma}}$ is so complicated that we will present its derivation in detail.

First, with the definition $\varphi=\xi \Phi^{\dagger} \Phi$, let us consider the non-minimal term

$$
\mathcal{L}_{N M}=\sqrt{-g} \varphi R=\mathcal{L}_{1}+\mathcal{L}_{2},
$$

where we have defined

$$
\begin{aligned}
\mathcal{L}_{1} & =\sqrt{-g} \varphi g^{\mu \nu}\left(\partial_{\alpha} \Gamma_{\mu \nu}^{\alpha}-\partial_{\nu} \Gamma_{\mu \alpha}^{\alpha}\right) \\
\mathcal{L}_{2} & =\sqrt{-g} \varphi g^{\mu \nu}\left(\Gamma_{\sigma \alpha}^{\alpha} \Gamma_{\mu \nu}^{\sigma}-\Gamma_{\sigma \nu}^{\alpha} \Gamma_{\mu \alpha}^{\sigma}\right)
\end{aligned}
$$

where as usual the affine connection and its contraction are defined as

$$
\Gamma_{\mu \nu}^{\alpha}=\frac{1}{2} g^{\alpha \beta}\left(\partial_{\mu} g_{\beta \nu}+\partial_{\nu} g_{\beta \mu}-\partial_{\beta} g_{\mu \nu}\right), \quad \Gamma_{\mu \alpha}^{\alpha}=\frac{1}{2} g^{\alpha \beta} \partial_{\mu} g_{\alpha \beta}=\frac{\partial_{\mu} \sqrt{-g}}{\sqrt{-g}} .
$$

$\mathcal{L}_{1}$ includes terms with second derivative of the metric, i.e., $\partial^{2} g$, so we need to perform the integration by parts to transform them to terms with first derivative, i.e., $\partial g$. After the integration by parts, $\mathcal{L}_{1}$ is devided in two parts, one of which contains terms proportional to $\partial \varphi$ and the other part does terms proportional to $\varphi$ itself

$\mathcal{L}_{1}=-\sqrt{-g} \partial_{\alpha} \varphi\left(g^{\mu \nu} \Gamma_{\mu \nu}^{\alpha}-g^{\alpha \mu} \Gamma_{\mu \beta}^{\beta}\right)-\varphi\left[\partial_{\alpha}\left(\sqrt{-g} g^{\mu \nu}\right) \Gamma_{\mu \nu}^{\alpha}-\partial_{\nu}\left(\sqrt{-g} g^{\mu \nu}\right) \Gamma_{\mu \alpha}^{\alpha}\right]$.

Now let us focus on the second term, which we call $A$, and show that $A$ is equal to $-2 \mathcal{L}_{2}$.

$$
\begin{aligned}
A & \equiv-\varphi\left[\partial_{\alpha}\left(\sqrt{-g} g^{\mu \nu}\right) \Gamma_{\mu \nu}^{\alpha}-\partial_{\nu}\left(\sqrt{-g} g^{\mu \nu}\right) \Gamma_{\mu \alpha}^{\alpha}\right] \\
& =-\varphi\left[\sqrt{-g}\left(\Gamma_{\alpha \beta}^{\beta} g^{\mu \nu}+\partial_{\alpha} g^{\mu \nu}\right) \Gamma_{\mu \nu}^{\alpha}-\sqrt{-g}\left(\Gamma_{\nu \beta}^{\beta} g^{\mu \nu}+\partial_{\nu} g^{\mu \nu}\right) \Gamma_{\mu \alpha}^{\alpha}\right]
\end{aligned}
$$

In terms of the definition of the affine connection (121), we can prove the following relations:

$$
\begin{aligned}
\partial_{\alpha} g^{\mu \nu} \Gamma_{\mu \nu}^{\alpha} & =-2 g^{\mu \nu} \Gamma_{\sigma \nu}^{\alpha} \Gamma_{\mu \alpha}^{\sigma}, \\
\partial_{\nu} g^{\mu \nu} & =-g^{\alpha \beta} \Gamma_{\alpha \beta}^{\mu}-g^{\mu \nu} \Gamma_{\nu \alpha}^{\alpha} .
\end{aligned}
$$

Inserting Eq. (124) to Eq. (123), we reach the result that $A$ is equal to $-2 \mathcal{L}_{2}$ :

$$
A=-2 \varphi \sqrt{-g} g^{\mu \nu}\left(\Gamma_{\sigma \alpha}^{\alpha} \Gamma_{\mu \nu}^{\sigma}-\Gamma_{\sigma \nu}^{\alpha} \Gamma_{\mu \alpha}^{\sigma}\right)=-2 \mathcal{L}_{2} .
$$


Next, plugging this result into Eq. (122) leads to

$$
\mathcal{L}_{1}=-\sqrt{-g} \partial_{\alpha} \varphi\left(g^{\mu \nu} \Gamma_{\mu \nu}^{\alpha}-g^{\alpha \mu} \Gamma_{\mu \beta}^{\beta}\right)-2 \mathcal{L}_{2} .
$$

Moreover, substituting Eq. (126) into Eq. (119), we have

$$
\begin{aligned}
\mathcal{L}_{N M} & =-\sqrt{-g} \partial_{\alpha} \varphi\left(g^{\mu \nu} \Gamma_{\mu \nu}^{\alpha}-g^{\alpha \mu} \Gamma_{\mu \beta}^{\beta}\right)-\mathcal{L}_{2} \\
& \equiv \mathcal{L}_{K}-\mathcal{L}_{2} .
\end{aligned}
$$

Here we have defined

$$
\begin{aligned}
\mathcal{L}_{K} & =-\sqrt{-g} \partial_{\alpha} \varphi\left(g^{\mu \nu} \Gamma_{\mu \nu}^{\alpha}-g^{\alpha \mu} \Gamma_{\mu \beta}^{\beta}\right) \\
& \equiv-\sqrt{-g} \partial_{\alpha} \varphi K^{\alpha}
\end{aligned}
$$

where $K^{\alpha}$ is defined as

$$
\begin{aligned}
K^{\alpha} & =g^{\mu \nu} \Gamma_{\mu \nu}^{\alpha}-g^{\alpha \mu} \Gamma_{\mu \beta}^{\beta} \\
& =\left(g^{\alpha \rho} g^{\mu \sigma}-g^{\alpha \mu} g^{\rho \sigma}\right) \partial_{\mu} g_{\rho \sigma}
\end{aligned}
$$

where at the second equality we have used Eqs. (121) and (124). With this expression (129), it is straightforward to take the variation of $\mathcal{L}_{K}$ with respect to $\partial_{\mu} g_{\rho \sigma}$ whose result is given by

$$
\frac{\partial \mathcal{L}_{K}}{\partial \partial_{\mu} g_{\rho \sigma}}=-\sqrt{-g} \partial_{\alpha} \varphi\left(g^{\alpha(\rho} g^{\sigma) \mu}-g^{\alpha \mu} g^{\rho \sigma}\right)
$$

Thus, we have

$$
\frac{\partial \mathcal{L}_{K}}{\partial \partial_{\mu} g_{\rho \sigma}} 2 g_{\rho \sigma}=6 \sqrt{-g} \partial^{\mu} \varphi
$$

Taking the variation of $\mathcal{L}_{2}$ with respect to $\partial_{\mu} g_{\rho \sigma}$ is a bit tedious but straightforward since the whole calculation can be performed by using the formula

$$
\frac{\partial \Gamma_{\alpha \beta}^{\lambda}}{\partial \partial_{\mu} g_{\rho \sigma}}=\frac{1}{2}\left[g^{\lambda \rho} \delta_{(\alpha}^{\mu} \delta_{\beta)}^{\sigma}+g^{\lambda \sigma} \delta_{(\alpha}^{\mu} \delta_{\beta)}^{\rho}-g^{\lambda \mu} \delta_{(\alpha}^{\rho} \delta_{\beta)}^{\sigma}\right]
$$

After a straightforward calculation using Eq. (132), we have the result

$$
\frac{\partial \mathcal{L}_{2}}{\partial \partial_{\mu} g_{\rho \sigma}}=\sqrt{-g} \varphi\left[\frac{1}{2} g^{\rho \sigma} g^{\alpha \beta} \Gamma_{\alpha \beta}^{\mu}-g^{\rho \alpha} g^{\sigma \beta} \Gamma_{\alpha \beta}^{\mu}+\frac{1}{2}\left(g^{\mu \rho} g^{\nu \sigma}+g^{\mu \sigma} g^{\nu \rho}-g^{\mu \nu} g^{\rho \sigma}\right) \Gamma_{\nu \alpha}^{\alpha}\right\}
$$

Hence, we obtain

$$
\frac{\partial \mathcal{L}_{2}}{\partial \partial_{\mu} g_{\rho \sigma}} 2 g_{\rho \sigma}=2 \sqrt{-g} \varphi K^{\mu}
$$


Accordingly, Eqs. (131) and (134) give us

$$
\frac{\partial \mathcal{L}_{N M}}{\partial \partial_{\mu} g_{\rho \sigma}} 2 g_{\rho \sigma}=2 \sqrt{-g}\left(3 \partial^{\mu} \varphi-\varphi K^{\mu}\right) .
$$

Since $\partial_{\mu} g_{\rho \sigma}$ is only included in $\mathcal{L}_{N M}$, from the definition $\varphi=\xi \Phi^{\dagger} \Phi$, we have

$$
\frac{\partial \mathcal{L}}{\partial \partial_{\mu} g_{\rho \sigma}} 2 g_{\rho \sigma}=2 \xi \sqrt{-g}\left[3 g^{\mu \nu} \partial_{\nu}\left(\Phi^{\dagger} \Phi\right)-\Phi^{\dagger} \Phi K^{\mu}\right] .
$$

Furthermore, it is easy to calculate the variation of the Lagrangian with respect to $\partial_{\mu} \Phi, \partial_{\mu} \Phi^{\dagger}$. The results read

$$
\begin{aligned}
\frac{\partial \mathcal{L}}{\partial \partial_{\mu} \Phi} \Phi & =-\sqrt{-g}\left[\xi \Phi^{\dagger} \Phi K^{\mu}+g^{\mu \nu}\left(D_{\nu} \Phi\right)^{\dagger} \Phi\right] \\
\frac{\partial \mathcal{L}}{\partial \partial_{\mu} \Phi^{\dagger}} \Phi^{\dagger} & =-\sqrt{-g}\left[\xi \Phi^{\dagger} \Phi K^{\mu}+g^{\mu \nu}\left(D_{\nu} \Phi\right) \Phi^{\dagger}\right] .
\end{aligned}
$$

Putting together Eqs. (136) and (137), the dilatation current (118) is calculated to be

$$
J^{\mu}=(6 \xi+1) \sqrt{-g} g^{\mu \nu} \partial_{\nu}\left(\Phi^{\dagger} \Phi\right)
$$

\section{B Useful formulae in the loop calculation}

In Appendix B, we summarize useful formulae in calculating radiative corrections in Section 5. Following Ref. [15], as a regularization method, we adopt the method of continuous space-time dimensions $D$ in a flat Minkowski space-time. In this regularization method, all the quantities are extended from four dimensions to $D$ dimensions. Let us therefore focus on the following loop integral:

$$
I(m, n)=\int d^{D} k \frac{\left(k^{2}\right)^{m-2}}{\left(k^{2}+\Delta\right)^{n}}
$$

where $m, n$ are integers and $\Delta$ is a constant. By power counting, this integral is convergent as long as $D<2 n-2 m+4$.

With a Wick rotation $k^{0}=i k^{D}$ and the ansatz of spherical symmetry, the integral (139) can be rewritten as

$$
I(m, n)=i V(D) \int_{0}^{\infty} d k \frac{k^{2 m+D-5}}{\left(k^{2}+\Delta\right)^{n}}
$$


where $V(D)=\frac{2 \pi^{\frac{D}{2}}}{\Gamma\left(\frac{D}{2}\right)}$ is a D-dimensional volume form, e.g., $V(4)=2 \pi^{2}$. Via the change of variables from $k$ to $t=\frac{k^{2}}{\Delta}$, the integral is reduced to

$$
\begin{aligned}
I(m, n) & =i V(D) \frac{1}{2} \Delta^{m-n+\frac{D}{2}-2} \int_{0}^{\infty} d t \frac{t^{m+\frac{D}{2}-3}}{(1+t)^{n}} \\
& =i V(D) \frac{1}{2} \Delta^{m-n+\frac{D}{2}-2} B\left(m+\frac{D}{2}-2, n-m-\frac{D}{2}+2\right),
\end{aligned}
$$

where the definition of the beta function is used:

$$
B(\alpha, \beta)=\int_{0}^{1} d x x^{\alpha-1}(1-x)^{\beta-1}=\int_{0}^{\infty} d t t^{\alpha-1}(1+t)^{-\alpha-\beta}=\frac{\Gamma(\alpha) \Gamma(\beta)}{\Gamma(\alpha+\beta)} .
$$

Since $\Gamma(z)$ has isolated poles at $z=0,-1,-2, \cdots$, the integral (141) has isolated poles at $D=2(n-m+2), 2(n-m+3), \cdots$. We often make use of a relation for the gamma function, which holds for positive real numbers $x>0$

$$
\Gamma(x+1)=x \Gamma(x)
$$

and $\Gamma(1)=1$.

In Section 5, to combine propagator denominators we utilize the Feyman parameter formula

$$
\frac{1}{A_{1} A_{2} \cdots A_{n}}=\int_{0}^{1} d x_{1} \cdots d x_{n} \delta\left(\sum x_{i}-1\right) \frac{(n-1) !}{\left(x_{1} A_{1}+x_{2} A_{2}+\cdots+x_{n} A_{n}\right)^{n}} .
$$

In the case of only two denominator factors, this formula reduces to

$$
\frac{1}{A B}=\int_{0}^{1} d x \frac{1}{[x A+(1-x) B]^{2}} .
$$

Taking differentiation of Eq. (145) with respect to $B$, we can derive another formula

$$
\frac{1}{A B^{2}}=\int_{0}^{1} d x \frac{2(1-x)}{[x A+(1-x) B]^{3}}
$$

\section{References}

[1] J. Iliopoulos, arXiv:1305.6779 [hep-ph].

[2] V. I. Ginzburg and L. D. Landau, Zh. Eksp. Teor. Fiz. 20 (1950) 1064 [in Russian].

[3] S. Coleman and J. Mandula, Phys. Rev. 159 (1967) 1251. 
[4] G. Aad et al. [ATLAS Collaboration], Phys. Lett. B 716 (2012) 1, arXiv:1207.7214 [hep-ex].

[5] S. Chatrchyan et al. [CMS Collaboration], Phys. Lett. B 716 (2012) 30, arXiv:1207.7235 [hep-ex].

[6] W. A. Bardeen, FERMILAB-CONF-95-391-T.

[7] K. A. Meissner and H. Nicolai, Phys. Lett. B 648 (2007) 312, arXiv:hepth/0612165; Phys. Lett. B 660 (2008) 260, arXiv:0710.2840 [hep-th].

[8] S. Iso, N. Okada and Y. Orisaka, Phys. Lett. B 676 (2009) 81, arXiv:0902.4050 [hep-ph]; Phys. Rev. D 80 (2009) 115007, arXiv:0909.0128 [hep-ph]; S. Iso and Y. Orisaka, PTEP 2013 (2013) 023B08, arXiv:1210.2848 [hep-ph].

[9] P. H. Frampton and C. Vafa, hep-th/9903226.

[10] G. 'tHooft, in Proc. of 1979 Cargese Institute on Recent Developments in Gauge Theories, p. 135, Plenum Press, New York, 1980.

[11] I. Oda, Phys. Rev. D 87 (2013) 065025, arXiv:1301.2709 [hep-ph].

[12] I. Oda, Phys. Lett. B 724 (2013) 160, arXiv:1305.0884 [hep-ph].

[13] S. R. Coleman and E. J. Weinberg, Phys. Rev. D 7 (1973) 1888.

[14] C. W. Misner, K. S. Thorne and J. A. Wheeler, "Gravitation", W H Freeman and Co (Sd), 1973.

[15] Y. Fujii and K. Maeda, "The Scalar-Tensor Theory of Gravitation", Cambridge University Press, 2003.

[16] I. Oda, Adv. Studies Theor. Phys. 2 (2008) 261, arXiv:0709.2419 [hep-th]; Mod. Phys. Lett. A 25 (2010) 2411, arXiv:1003.1437 [hep-th]; Phys. Lett. B 690 (2010) 322, arXiv:1004.3078 [hep-th].

[17] Y. Fujii, Prog. Theor. Phys. 99 (1998) 599.

[18] F. L. Bezrukov and M. Shaposhnikov, Phys. Lett. B 659 (2008) 703, arXiv:0710.3755 [hep-th]; F. L. Bezrukov, arXiv:1307.0708 [hep-ph], references therein.

Received: January 11, 2014 